\title{
Geometric and Energetic Aspects of Aluminum Nitride Cages
}

\author{
Hai-Shun Wu, ${ }^{a,}$ Fu-Qiang Zhang, ${ }^{a}$ Xiao-Hong Xu, ${ }^{a}$ Cong-Jie Zhang, ${ }^{a}$ Haijun Jiao ${ }^{b, a},{ }^{*}$
}

a) Department of Chemistry, Shanxi Normal University, Linfen, 041004. China. b) Institut für

Organische Katalyseforschung (IfOK) an der Universität Rostock e.V. Buchbinderstrasse 5-6, 18055

Rostock, Germany

hjiao@ifok.uni-rostock.de 
Table S1. Computed Total Electronic Energies (au) for the most stable $(\mathrm{AlN})_{\mathrm{n}}(n=2-41)$ structures.

\begin{tabular}{|c|c|c|c|c|}
\hline$(\mathrm{AlN})_{\mathrm{n}} /$ Symmetry & ZPE (NImag) ${ }^{a}$ & $\mathrm{E}_{\mathrm{tot}} \mathrm{a}^{\mathrm{a}}$ & $\mathrm{E}_{\mathrm{tot}} \mathrm{b}$ & $\mathrm{E}_{\mathrm{tot}}$ \\
\hline \multirow[t]{2}{*}{$(\mathrm{AlN})_{1} / C \cdot \cdot_{\mathrm{v}}$} & $0.9(0)$ & $-56.26206(\mathrm{~T})$ & $-56.61607(\mathrm{~T})$ & $(-297.04621)^{\mathrm{c}, \mathrm{d}}$ \\
\hline & $1.0(0)$ & $-56.15291(\mathrm{~S})$ & $-56.57389(\mathrm{~S})$ & $(-297.00339)^{\mathrm{c}, \mathrm{d}}$ \\
\hline$(\mathrm{AlN})_{2} / D_{2 \mathrm{~h}}$ & $4.5(0)$ & -112.63462 & -113.48523 & \\
\hline$(\mathrm{AlN})_{3} / D_{3 \mathrm{~h}}$ & $9.7(0)$ & -168.90204 & -170.23156 & \\
\hline$(\mathrm{AlN})_{4} / D_{4 \mathrm{~h}}$ & $14.3(0)$ & -225.33924 & -227.06667 & \\
\hline$(\mathrm{AlN})_{5} / D_{5 \mathrm{~h}}$ & $18.6(0)$ & -281.75333 & -283.87899 & \\
\hline$(\mathrm{AlN})_{6} / D_{3 \mathrm{~d}}$ & $24.7(0)$ & -338.19299 & -340.80278 & \\
\hline$(\mathrm{AlN})_{7} / C_{3 \mathrm{v}}$ & $28.9(0)$ & -394.56132 & -397.60304 & \\
\hline$(\mathrm{AlN})_{8} / S_{4}$ & $34.1(0)$ & -451.09176 & -454.52967 & \\
\hline$(\mathrm{AlN})_{9} / C_{3 \mathrm{~h}}$ & $39.1(0)$ & -507.56768 & -511.41541 & \\
\hline$(\mathrm{AlN})_{10} / C_{3}$ & $43.4(0)$ & -563.97873 & -568.24845 & \\
\hline$(\mathrm{AlN})_{11} / C_{\mathrm{s}}$ & $48.5(0)$ & -620.49450 & -625.16029 & $(-3269.87013)^{\mathrm{c}, \mathrm{d}}$ \\
\hline$(\mathrm{AlN})_{12} / T_{\mathrm{h}}$ & $53.8(0)$ & -677.05454 & -682.10573 & $(-3567.24299)^{\mathrm{c}, \mathrm{d}}$ \\
\hline$(\mathrm{AlN})_{12} / D_{3 \mathrm{~h}}$ & & -676.59970 & -681.71298 & \\
\hline$(\mathrm{AlN})_{12} / T_{\mathrm{h}}$ & & -676.48020 & -681.45933 & \\
\hline$(\mathrm{AlN})_{13} / C_{1}$ & $57.7(0)$ & -733.39004 & -738.88592 & $(-3864.44704)^{\mathrm{c}, \mathrm{d}}$ \\
\hline$(\mathrm{AlN})_{14} / C_{\mathrm{s}}$ & $62.7(0)$ & -789.89827 & -795.79336 & \\
\hline$(\mathrm{AlN})_{15} / C_{3 \mathrm{~h}}$ & $67.8(0)$ & -846.42741 & -852.71471 & \\
\hline$(\mathrm{AlN})_{16} / T_{\mathrm{d}}{ }^{\mathrm{a}}$ & $72.7(0)$ & -902.91046 & -909.60755 & $(-4756.58729)^{\mathrm{e}}$ \\
\hline$(\mathrm{AlN})_{16} / C_{3}$ & & -902.72807 & -909.47310 & $(-4756.45839)^{\mathrm{e}}$ \\
\hline$(\mathrm{AlN})_{16} / C_{3 \mathrm{v}}$ & & -902.88284 & -909.58101 & $(-4756.56973)^{\mathrm{e}}$ \\
\hline$(\mathrm{AlN})_{16} / S_{4}$ & & -902.62743 & -909.39289 & $(-4756.38750)^{\mathrm{e}}$ \\
\hline$(\mathrm{AlN})_{17} / C_{\mathrm{s}}$ & $76.9(0)$ & -959.30793 & -966.42757 & \\
\hline$(\mathrm{AlN})_{18} / S_{6}$ & $81.8(0)$ & -1015.80590 & -1023.32481 & \\
\hline$(\mathrm{AlN})_{19} / C_{3}$ & $86.2(0)$ & -1072.21756 & -1080.16487 & \\
\hline$(\mathrm{AlN})_{20} / C_{2}$ & $90.9(0)$ & -1128.69036 & -1137.04750 & \\
\hline$(\mathrm{AlN})_{21} / C_{3 \mathrm{~h}}$ & $95.8(0)$ & -1185.19505 & -1193.94042 & \\
\hline$(\mathrm{AlN})_{22} / C_{3}$ & $100.7(0)$ & -1241.67077 & -1250.83119 & \\
\hline
\end{tabular}




\begin{tabular}{|c|c|c|c|}
\hline$(\mathrm{AlN})_{23} / C_{1}$ & $105.2(0)$ & -1298.11446 & -1307.67118 \\
\hline$(\mathrm{AlN})_{24} / S_{4}$ & $109.9(0)$ & -1354.59354 & -1364.56658 \\
\hline$(\mathrm{AlN})_{25} / C_{3}$ & $114.7(0)$ & -1411.07574 & -1421.47327 \\
\hline$(\mathrm{AlN})_{26} / C_{2 \mathrm{~h}}$ & $119.3(0)$ & -1467.52924 & -1478.33367 \\
\hline$(\mathrm{AlN})_{27} / C_{3 \mathrm{v}}$ & $124.1(0)$ & -1524.00271 & -1535.23063 \\
\hline$(\mathrm{AlN})_{28} / T$ & $128.9(0)$ & -1580.50662 & -1592.10508 \\
\hline$(\mathrm{AlN})_{29} / C_{\mathrm{s}}$ & & -1636.94095 & -1648.98617 \\
\hline$(\mathrm{AlN})_{30} / C_{2}$ & & -1693.38704 & -1705.86240 \\
\hline$(\mathrm{AlN})_{31} / C_{3}$ & & -1749.90089 & -1762.77067 \\
\hline$(\mathrm{AlN})_{32} / C_{1}$ & & -1806.34569 & \\
\hline$(\mathrm{AlN})_{33} / C_{\mathrm{s}}$ & & -1862.81416 & \\
\hline$(\mathrm{AlN})_{34} / C_{3}$ & & -1919.31463 & \\
\hline$(\mathrm{AlN})_{35} / C_{\mathrm{s}}$ & & -1975.78539 & \\
\hline$(\mathrm{AlN})_{36} / T_{\mathrm{d}}$ & & -2032.25676 & \\
\hline$(\mathrm{AlN})_{36} / C_{3 \mathrm{~h}}$ & & -2032.26335 & \\
\hline$(\mathrm{AlN})_{36} / C_{3}$ & & -2032.25401 & \\
\hline$(\mathrm{AlN})_{36} / S_{6}$ & & -2032.25308 & \\
\hline$(\mathrm{AlN})_{36} / C_{2}$ & & -2032.25388 & \\
\hline$(\mathrm{AlN})_{36} / C_{1}$ & & -2032.24631 & \\
\hline$(\mathrm{AlN})_{37} / C_{\mathrm{s}}$ & & -2088.75039 & \\
\hline$(\mathrm{AlN})_{38} / C_{2}$ & & -2145.20383 & \\
\hline$(\mathrm{AlN})_{39} / C_{1}$ & & -2201.67482 & \\
\hline$(\mathrm{AlN})_{40} / C_{3}$ & & -2258.15408 & \\
\hline$(\mathrm{AlN})_{41} / C_{1}$ & & -2314.63355 & \\
\hline
\end{tabular}

(a) At HF/LANL2DZ. (b) At B3LYP/LANL2DZp//LANL2DZ. (c) At B3LYP/6-31G*. (d) At the B3LYP/6-311G*//B3LYP/6-31G* level, -297.07939/(AlN)/triplet; -297.03755 /(AlN) ${ }_{1} /$ singlet; $-3270.23039 /(\mathrm{AlN})_{11} ;-3567.63793 /(\mathrm{AlN})_{12},-3864.87099 /(\mathrm{AlN})_{13}$ (e) At B3LYP/cc-pvDZ. 
HF / LANL2DZ / / Fopt

$\begin{array}{rrrr}(\mathrm{AlN})_{2} / \mathrm{D}_{2 \mathrm{~h}} & & \\ \mathrm{Al} & 1.97514619 & 0.00000000 & 0.00000000 \\ \mathrm{Al} & -1.97514619 & 0.00000000 & 0.00000000 \\ \mathrm{~N} & 0.00000000 & 0.64540328 & 0.00000000 \\ \mathrm{~N} & 0.00000000 & -0.64540328 & 0.00000000\end{array}$

\section{(AlN) ${ }_{3} / D_{3 \mathrm{~h}}$}

$\begin{array}{rrrr}\text { Al } & -1.55679933 & 0.00000000 & -0.41714312 \\ \mathrm{~N} & -1.31974799 & 0.00000000 & 1.31974799 \\ \text { Al } & 0.41714312 & 0.00000000 & 1.55679933 \\ \mathrm{~N} & 1.80280927 & 0.00000000 & 0.48306129 \\ \mathrm{Al} & 1.13965620 & 0.00000000 & -1.13965620 \\ \mathrm{~N} & -0.48306129 & 0.00000000 & -1.80280927\end{array}$

\section{$(\mathrm{AlN})_{4} / D_{4 \mathrm{~h}}$}

$\begin{array}{rrrr}\mathrm{N} & -1.84829599 & -0.00000002 & -1.41824739 \\ \mathrm{Al} & -2.11715721 & -0.00000000 & 0.27872904 \\ \mathrm{Al} & 2.11715721 & 0.00000000 & -0.27872904 \\ \mathrm{~N} & -1.41824739 & 0.00000000 & 1.84829599 \\ \mathrm{~N} & 1.84829599 & 0.00000002 & 1.41824739 \\ \mathrm{Al} & 0.27872904 & 0.00000002 & 2.11715721 \\ \mathrm{Al} & -0.27872904 & -0.00000002 & -2.11715721 \\ \mathrm{~N} & 1.41824739 & -0.00000000 & -1.84829599\end{array}$

\section{(AlN) ${ }_{5} / D_{5 \mathrm{~h}}$}

$\begin{array}{rrrr}\text { Al } & 1.57204761 & 2.16373791 & 0.00000000 \\ \text { Al } & 2.54362647 & -0.82647434 & 0.00000000 \\ \text { Al } & 0.00000000 & -2.67452715 & 0.00000000 \\ \text { Al } & -2.54362647 & -0.82647434 & 0.00000000 \\ \text { Al } & -1.57204761 & 2.16373791 & 0.00000000 \\ \text { N } & 0.00000000 & 2.82021079 & 0.00000000 \\ \text { N } & 2.68217985 & 0.87149306 & 0.00000000 \\ \text { N } & 1.65767831 & -2.28159846 & 0.00000000 \\ \text { N } & -1.65767831 & -2.28159846 & 0.00000000 \\ \text { N } & -2.68217985 & 0.87149306 & 0.00000000\end{array}$

\section{(AlN) ${ }_{6} / D_{3 \mathrm{~d}}$}

$\begin{array}{rrrr}\text { Al } & 1.78708744 & 0.00000000 & 0.94192101 \\ \mathrm{~N} & 1.87368877 & 0.00000000 & -0.99768846 \\ \mathrm{Al} & -0.89354372 & 1.54766313 & 0.94192101 \\ \mathrm{Al} & -0.89354372 & -1.54766313 & 0.94192101 \\ \mathrm{~N} & -0.93684438 & 1.62266207 & -0.99768846 \\ \mathrm{~N} & -0.93684438 & -1.62266207 & -0.99768846 \\ \mathrm{Al} & 0.89354372 & 1.54766313 & -0.94192101 \\ \mathrm{~N} & 0.93684438 & 1.62266207 & 0.99768846 \\ \mathrm{Al} & -1.78708744 & 0.00000000 & -0.94192101 \\ \mathrm{Al} & 0.89354372 & -1.54766313 & -0.94192101 \\ \mathrm{~N} & -1.87368877 & 0.00000000 & 0.99768846 \\ \mathrm{~N} & 0.93684438 & -1.62266207 & 0.99768846\end{array}$




\begin{tabular}{rrrr}
\multicolumn{2}{c}{$(\mathrm{AlN})_{7} / C_{3 \mathrm{v}}$} & & \\
$\mathrm{Al}$ & 0.00000000 & 1.74430188 & 0.92879762 \\
$\mathrm{~N}$ & -1.37736702 & 0.79522322 & 1.82217384 \\
$\mathrm{~N}$ & 0.00000000 & 1.79046017 & -0.87204433 \\
$\mathrm{Al}$ & -1.26183984 & 0.72852357 & -1.78360376 \\
$\mathrm{Al}$ & 0.00000000 & 0.00000000 & 2.80601777 \\
$\mathrm{~N}$ & 0.00000000 & 0.00000000 & -2.95393111 \\
$\mathrm{~N}$ & 1.37736702 & 0.79522322 & 1.82217384 \\
$\mathrm{Al}$ & 1.26183984 & 0.72852357 & -1.78360376 \\
$\mathrm{Al}$ & -1.51060974 & -0.87215094 & 0.92879762 \\
$\mathrm{Al}$ & 1.51060974 & -0.87215094 & 0.92879762 \\
$\mathrm{~N}$ & 0.00000000 & -1.59044643 & 1.82217384 \\
$\mathrm{~N}$ & -1.55058399 & -0.89523008 & -0.87204433 \\
$\mathrm{~N}$ & 1.55058399 & -0.89523008 & -0.87204433 \\
$\mathrm{Al}$ & 0.00000000 & -1.45704714 & -1.78360376
\end{tabular}

\section{(AlN) $8 / S_{4}$}

$\begin{array}{rr}\text { N } & 0.96827175 \\ \text { Al } & 0.91971570 \\ N & 1.67646724 \\ \text { Al } & 0.85338048 \\ N & -0.96827175 \\ N & -0.97784734 \\ N & 0.97784734 \\ \text { Al } & -0.91971570 \\ \text { Al } & -1.69277470 \\ \text { Al } & 1.69277470 \\ N & -1.67646724 \\ N & -1.00712597 \\ N & 1.00712597 \\ \text { Al } & -0.85338048 \\ \text { Al } & -0.93791615 \\ \text { Al } & 0.93791615\end{array}$

\begin{tabular}{rr}
0.97784734 & \multicolumn{1}{c}{-2.45642403} \\
1.69277470 & -0.75944456 \\
1.00712597 & 0.72748489 \\
0.93791615 & 2.36280367 \\
-0.97784734 & -2.45642403 \\
0.96827175 & 2.45642403 \\
-0.96827175 & 2.45642403 \\
-1.69277470 & -0.75944456 \\
0.91971570 & 0.75944456 \\
-0.91971570 & 0.75944456 \\
-1.00712597 & 0.72748489 \\
1.67646724 & -0.72748489 \\
-1.67646724 & -0.72748489 \\
-0.93791615 & 2.36280367 \\
0.85338048 & -2.36280367 \\
-0.85338048 & -2.36280367
\end{tabular}

\section{(AlN) $9 / C_{3 \mathrm{~h}}$}

$\begin{array}{rrrr}\text { Al } & 0.01941678 & 1.85336224 & 1.56090234 \\ \mathrm{~N} & 1.62905810 & 0.85669486 & 1.61484710 \\ \mathrm{~N} & 0.50412600 & 2.72792255 & 0.00000000 \\ \mathrm{Al} & 2.03161309 & 1.65893911 & 0.00000000 \\ \mathrm{Al} & -1.61476717 & -0.90986570 & 1.56090234 \\ \mathrm{Al} & 1.59535040 & -0.94349655 & 1.56090234 \\ \mathrm{~N} & -1.55644856 & 0.98245827 & 1.61484710 \\ \mathrm{~N} & -0.07260954 & -1.83915313 & 1.61484710 \\ \mathrm{~N} & -2.61451323 & -0.92737536 & 0.00000000 \\ \mathrm{~N} & 2.11038723 & -1.80054720 & 0.00000000 \\ \mathrm{Al} & -2.45248996 & 0.92995900 & 0.00000000 \\ \mathrm{Al} & 0.42087686 & -2.58889810 & 0.00000000 \\ \mathrm{Al} & 0.01941678 & 1.85336224 & -1.56090234 \\ \mathrm{~N} & 1.62905810 & 0.85669486 & -1.61484710 \\ \mathrm{Al} & -1.61476717 & -0.90986570 & -1.56090234 \\ \mathrm{Al} & 1.59535040 & -0.94349655 & -1.56090234 \\ \mathrm{~N} & -1.55644856 & 0.98245827 & -1.61484710\end{array}$




\begin{tabular}{rr}
$(\mathrm{AlN})$ & \multicolumn{1}{l}{$/ C_{3}$} \\
$\mathrm{~N}$ & 0.00027433 \\
$\mathrm{~N}$ & -0.35631466 \\
$\mathrm{Al}$ & 0.60100794 \\
$\mathrm{Al}$ & 2.65281821 \\
$\mathrm{~N}$ & 2.21315959 \\
$\mathrm{Al}$ & 1.15238135 \\
$\mathrm{~N}$ & 1.44543982 \\
$\mathrm{Al}$ & -0.00029188 \\
$\mathrm{~N}$ & -2.19940618 \\
$\mathrm{~N}$ & 2.55585201 \\
$\mathrm{Al}$ & -1.79751381 \\
$\mathrm{Al}$ & 1.19717652 \\
$\mathrm{Al}$ & -1.84334995 \\
$\mathrm{Al}$ & -0.80958835 \\
$\mathrm{~N}$ & -2.86203906 \\
$\mathrm{~N}$ & 0.64918917 \\
$\mathrm{Al}$ & -2.83876335 \\
$\mathrm{Al}$ & 1.68609106 \\
$\mathrm{~N}$ & -1.66815037 \\
$\mathrm{~N}$ & 0.22200918
\end{tabular}

$\begin{array}{rr}-0.00001422 & -1.97637119 \\ 2.74544782 & -0.31475997 \\ 1.72897534 & -1.60491579 \\ 0.59684393 & 0.29560860 \\ 2.02719995 & -0.74943202 \\ 2.61242719 & 0.69006180 \\ 1.09129415 & 1.68895977 \\ 0.00001350 & 2.10056059 \\ -1.68134026 & -0.31498655 \\ -1.06411140 & -0.31432663 \\ -0.34420539 & -1.60523411 \\ -1.38479694 & -1.60480749 \\ 1.99902207 & 0.29497266 \\ -2.59585684 & 0.29514964 \\ 0.90295718 & -0.75013224 \\ -2.93017188 & -0.74961374 \\ -0.30813283 & 0.68952556 \\ -2.30428221 & 0.69017304 \\ 0.70635621 & 1.68852547 \\ -1.79762091 & 1.68881088\end{array}$

\section{(AlN) ${ }_{11} / C_{\mathrm{s}}$}

$\begin{array}{rr}\mathrm{N} & 0.00000000 \\ \mathrm{Al} & 0.00000000 \\ \mathrm{Al} & 1.56599415 \\ \mathrm{~N} & 1.33670464 \\ \mathrm{Al} & 2.80674166 \\ \mathrm{~N} & 3.12902210 \\ \mathrm{Al} & 0.00000000 \\ \mathrm{~N} & 1.54202672 \\ \mathrm{Al} & 0.00000000 \\ \mathrm{Al} & 3.01275469 \\ \mathrm{~N} & 0.00000000 \\ \mathrm{Al} & 1.29498832 \\ \mathrm{~N} & 0.00000000 \\ \mathrm{~N} & 2.82859358 \\ \mathrm{Al} & -1.56599415 \\ \mathrm{~N} & -1.33670464 \\ \mathrm{Al} & -2.80674166 \\ \mathrm{~N} & -3.12902210 \\ \mathrm{~N} & -1.54202672 \\ \mathrm{Al} & -3.01275469 \\ \mathrm{Al} & -1.29498832 \\ \mathrm{~N} & -2.82859358\end{array}$

(AlN $)_{12} / T_{\mathrm{h}}$

$\begin{array}{rlll}\mathrm{N} & 1.33797030 & 0.00000000 & 2.60070729 \\ \mathrm{Al} & 2.55991123 & 0.00000000 & 1.29251276 \\ \mathrm{~N} & 2.60070729 & 1.33797030 & 0.00000000\end{array}$




$\begin{array}{rrrr}\mathrm{N} & 0.00000000 & 2.60070729 & 1.33797030 \\ \mathrm{Al} & 1.29251276 & 2.55991123 & 0.00000000 \\ \mathrm{Al} & 0.00000000 & 1.29251276 & 2.55991123 \\ \mathrm{~N} & 1.33797030 & 0.00000000 & -2.60070729 \\ \mathrm{Al} & 2.55991123 & 0.00000000 & -1.29251276 \\ \mathrm{~N} & 0.00000000 & 2.60070729 & -1.33797030 \\ \mathrm{Al} & 0.00000000 & 1.29251276 & -2.55991123 \\ \mathrm{~N} & 2.60070729 & -1.33797030 & 0.00000000 \\ \mathrm{~N} & 0.00000000 & -2.60070729 & 1.33797030 \\ \mathrm{Al} & 1.29251276 & -2.55991123 & 0.00000000 \\ \mathrm{Al} & 0.00000000 & -1.29251276 & 2.55991123 \\ \mathrm{~N} & 0.00000000 & -2.60070729 & -1.33797030 \\ \mathrm{Al} & 0.00000000 & -1.29251276 & -2.55991123 \\ \mathrm{~N} & -1.33797030 & 0.00000000 & 2.60070729 \\ \mathrm{Al} & -2.55991123 & 0.00000000 & 1.29251276 \\ \mathrm{~N} & -2.60070729 & 1.33797030 & 0.00000000 \\ \mathrm{Al} & -1.29251276 & 2.55991123 & 0.00000000 \\ \mathrm{~N} & -1.33797030 & 0.00000000 & -2.60070729 \\ \mathrm{Al} & -2.55991123 & 0.00000000 & -1.29251276 \\ \mathrm{~N} & -2.60070729 & -1.33797030 & 0.00000000 \\ \mathrm{Al} & -1.29251276 & -2.55991123 & 0.00000000\end{array}$

\begin{tabular}{rrrr}
\multicolumn{3}{c}{$(\mathrm{AlN})_{13} / C_{1}$} & \\
$\mathrm{~N}$ & 0.21241702 & 1.76297486 & 2.01544452 \\
$\mathrm{Al}$ & -0.38227480 & 2.58882871 & 0.46879453 \\
$\mathrm{~N}$ & 0.45891667 & 2.13761017 & -1.07289594 \\
$\mathrm{Al}$ & 2.24576668 & 1.94775958 & -0.94448974 \\
$\mathrm{~N}$ & 2.98204069 & 1.38719485 & 0.70771876 \\
$\mathrm{Al}$ & 1.79689157 & 0.88009184 & 2.01743517 \\
$\mathrm{Al}$ & -0.44112673 & 0.78398235 & -2.00965503 \\
$\mathrm{~N}$ & -2.25592099 & 0.81484824 & -2.00924626 \\
$\mathrm{Al}$ & -3.17087106 & 1.46389380 & -0.47568531 \\
$\mathrm{~N}$ & -2.13611854 & 2.17144870 & 0.78562710 \\
$\mathrm{~N}$ & 3.16973959 & 0.71517109 & -1.93009471 \\
$\mathrm{Al}$ & 2.23253845 & -0.87218732 & -1.94014476 \\
$\mathrm{~N}$ & 0.41358535 & -0.89645804 & -1.93011323 \\
$\mathrm{Al}$ & -1.48749194 & 1.06962912 & 2.13919391 \\
$\mathrm{Al}$ & 3.87660231 & 0.12813279 & -0.28673737 \\
$\mathrm{~N}$ & -4.04261351 & -0.12813291 & -0.18626063 \\
$\mathrm{Al}$ & -3.13357472 & -0.76519924 & -1.70738363 \\
$\mathrm{~N}$ & 3.17861535 & -1.55812126 & -0.44053401 \\
$\mathrm{~N}$ & 1.57683840 & -0.92213884 & 2.24828470 \\
$\mathrm{Al}$ & 2.10735542 & -2.08712183 & 0.88880564 \\
$\mathrm{Al}$ & -0.12598262 & -1.61216415 & 2.03273586 \\
$\mathrm{~N}$ & 0.39625943 & -2.66681194 & 0.59865214 \\
$\mathrm{Al}$ & -2.96406930 & -1.29380911 & 0.75877730 \\
$\mathrm{~N}$ & -2.25509693 & -2.07270423 & -0.80855130 \\
$\mathrm{Al}$ & -0.46643471 & -2.22680093 & -0.92760576 \\
$\mathrm{~N}$ & -1.73608905 & -0.74703882 & 2.01595139
\end{tabular}

(AlN) ${ }_{14} / C_{\mathrm{s}}$

Al $\quad 0.00000000 \quad-1.31220127 \quad-2.43683734$ 


$\begin{array}{rrrr}\mathrm{N} & 0.00000000 & 0.51147401 & -2.28967300 \\ \mathrm{~N} & 1.32973535 & -2.45308024 & -1.80069802 \\ \mathrm{Al} & 1.61869112 & 1.29615961 & -1.72492444 \\ \mathrm{~N} & 2.79020175 & 0.15066658 & -0.96127436 \\ \mathrm{Al} & 2.66715398 & -1.67210321 & -0.92701449 \\ \mathrm{~N} & 1.54849311 & 2.93536216 & -0.94680843 \\ \mathrm{Al} & 0.00000000 & 3.88042569 & -0.85726781 \\ \mathrm{Al} & 0.00000000 & -3.40190201 & -0.92436005 \\ \mathrm{~N} & 0.00000000 & -1.33623228 & 2.48410426 \\ \mathrm{Al} & 0.00000000 & 0.46566303 & 2.33758151 \\ \mathrm{Al} & 1.28588378 & -2.44965748 & 1.73763395 \\ \mathrm{~N} & 1.58012487 & 1.26079281 & 1.71855703 \\ \mathrm{Al} & 2.71414048 & 0.10792877 & 0.88908781 \\ \mathrm{~N} & 2.70743607 & -1.73015156 & 0.94331383 \\ \mathrm{Al} & 1.47785358 & 2.90308538 & 0.94771739 \\ \mathrm{~N} & 0.00000000 & 3.98158828 & 1.03564183 \\ \mathrm{~N} & 0.00000000 & -3.48521448 & 0.87912536 \\ \mathrm{~N} & -1.32973535 & -2.45308024 & -1.80069802 \\ \mathrm{Al} & -1.61869112 & 1.29615961 & -1.72492444 \\ \mathrm{~N} & -2.79020175 & 0.15066658 & -0.96127436 \\ \mathrm{Al} & -2.66715398 & -1.67210321 & -0.92701449 \\ \mathrm{~N} & -1.54849311 & 2.93536216 & -0.94680843 \\ \mathrm{Al} & -1.28588378 & -2.44965748 & 1.73763395 \\ \mathrm{~N} & -1.58012487 & 1.26079281 & 1.71855703 \\ \mathrm{Al} & -2.71414048 & 0.10792877 & 0.88908781 \\ \mathrm{~N} & -2.70743607 & -1.73015156 & 0.94331383 \\ \mathrm{Al} & -1.47785358 & 2.90308538 & 0.94771739\end{array}$

\begin{tabular}{|c|c|c|c|}
\hline \multicolumn{4}{|c|}{$(\mathrm{AlN})_{15} / C_{3 \mathrm{~h}}$} \\
\hline Al & 1.88704521 & 1.84488405 & 1.52274978 \\
\hline $\mathrm{N}$ & 0.24758011 & 2.71039212 & 1.52808929 \\
\hline $\mathrm{N}$ & 2.55762736 & 1.11281684 & 0.00000000 \\
\hline Al & -0.71107625 & 2.74336168 & 0.00000000 \\
\hline $\mathrm{N}$ & 1.53999013 & 1.00670341 & 3.13739500 \\
\hline Al & -0.14370782 & 1.80353524 & 3.07955739 \\
\hline Al & -2.54123906 & 0.71178706 & 1.52274978 \\
\hline Al & 0.65419385 & -2.55667112 & 1.52274978 \\
\hline $\mathrm{N}$ & -2.47105849 & -1.14078539 & 1.52808929 \\
\hline $\mathrm{N}$ & 2.22347838 & -1.56960673 & 1.52808929 \\
\hline $\mathrm{N}$ & -2.24254133 & 1.65856185 & 0.00000000 \\
\hline $\mathrm{N}$ & -0.31508603 & -2.77137869 & 0.00000000 \\
\hline Al & -2.02028278 & -1.98749094 & 0.00000000 \\
\hline Al & 2.73135903 & -0.75587074 & 0.00000000 \\
\hline $\mathrm{N}$ & -1.64182579 & 0.83031887 & 3.13739500 \\
\hline $\mathrm{N}$ & 0.10183566 & -1.83702228 & 3.13739500 \\
\hline Al & -1.49005343 & -1.02622225 & 3.07955739 \\
\hline Al & 1.63376125 & -0.77731300 & 3.07955739 \\
\hline Al & 1.88704521 & 1.84488405 & -1.52274978 \\
\hline $\mathrm{N}$ & 0.24758011 & 2.71039212 & -1.52808929 \\
\hline $\mathrm{N}$ & 1.53999013 & 1.00670341 & -3.13739500 \\
\hline Al & -0.14370782 & 1.80353524 & -3.07955739 \\
\hline Al & -2.54123906 & 0.71178706 & -1.52274978 \\
\hline
\end{tabular}




$\begin{array}{rrrr}\text { Al } & 0.65419385 & -2.55667112 & -1.52274978 \\ \mathrm{~N} & -2.47105849 & -1.14078539 & -1.52808929 \\ \mathrm{~N} & 2.22347838 & -1.56960673 & -1.52808929 \\ \mathrm{~N} & -1.64182579 & 0.83031887 & -3.13739500 \\ \mathrm{~N} & 0.10183566 & -1.83702228 & -3.13739500 \\ \mathrm{Al} & -1.49005343 & -1.02622225 & -3.07955739 \\ \mathrm{Al} & 1.63376125 & -0.77731300 & -3.07955739\end{array}$

\begin{tabular}{|c|c|c|c|}
\hline \multicolumn{4}{|c|}{$(\mathrm{AlN})_{16} / T_{\mathrm{d}}$} \\
\hline $\mathrm{N}$ & 0.94942717 & 3.10494149 & -0.94942717 \\
\hline Al & -0.89412050 & 3.05231693 & -0.89412050 \\
\hline $\mathrm{N}$ & -0.94942717 & 3.10494149 & 0.94942717 \\
\hline Al & 0.89412050 & 3.05231693 & 0.89412050 \\
\hline Al & 0.89412050 & -3.05231693 & -0.89412050 \\
\hline $\mathrm{N}$ & -0.94942717 & -3.10494149 & -0.94942717 \\
\hline $\mathrm{Al}$ & -0.89412050 & -3.05231693 & 0.89412050 \\
\hline $\mathrm{N}$ & 0.94942717 & -3.10494149 & 0.94942717 \\
\hline Al & 3.05231693 & -0.89412050 & -0.89412050 \\
\hline $\mathrm{N}$ & 3.10494149 & 0.94942717 & -0.94942717 \\
\hline Al & 3.05231693 & 0.89412050 & 0.89412050 \\
\hline $\mathrm{N}$ & 3.10494149 & -0.94942717 & 0.94942717 \\
\hline $\mathrm{N}$ & -3.10494149 & -0.94942717 & -0.94942717 \\
\hline Al & -3.05231693 & 0.89412050 & -0.89412050 \\
\hline $\mathrm{N}$ & -3.10494149 & 0.94942717 & 0.94942717 \\
\hline Al & -3.05231693 & -0.89412050 & 0.89412050 \\
\hline $\mathrm{N}$ & 0.94942717 & -0.94942717 & 3.10494149 \\
\hline Al & 0.89412050 & 0.89412050 & 3.05231693 \\
\hline $\mathrm{N}$ & -0.94942717 & 0.94942717 & 3.10494149 \\
\hline Al & -0.89412050 & -0.89412050 & 3.05231693 \\
\hline Al & 0.89412050 & -0.89412050 & -3.05231693 \\
\hline $\mathrm{N}$ & 0.94942717 & 0.94942717 & -3.10494149 \\
\hline Al & -0.89412050 & 0.89412050 & -3.05231693 \\
\hline $\mathrm{N}$ & -0.94942717 & -0.94942717 & -3.10494149 \\
\hline Al & 1.92189118 & -1.92189118 & 1.92189118 \\
\hline $\mathrm{N}$ & -1.88443385 & -1.88443385 & 1.88443385 \\
\hline Al & -1.92189118 & -1.92189118 & -1.92189118 \\
\hline $\mathrm{N}$ & 1.88443385 & -1.88443385 & -1.88443385 \\
\hline $\mathrm{N}$ & 1.88443385 & 1.88443385 & 1.88443385 \\
\hline Al & -1.92189118 & 1.92189118 & 1.92189118 \\
\hline $\mathrm{N}$ & -1.88443385 & 1.88443385 & -1.88443385 \\
\hline $\mathrm{Al}$ & 1.92189118 & 1.92189118 & -1.92189118 \\
\hline
\end{tabular}

\begin{tabular}{rrrr}
\multicolumn{4}{c}{$(\mathrm{AlN})_{17} / C_{\mathbf{s}}$} \\
$\mathrm{N}$ & 3.85768981 & 0.06077619 & 0.00000000 \\
$\mathrm{Al}$ & 3.40018564 & -1.61720538 & 0.00000000 \\
$\mathrm{~N}$ & 2.35964194 & -2.34144934 & -1.33926034 \\
$\mathrm{Al}$ & 1.26348115 & -1.23713554 & -2.29275237 \\
$\mathrm{~N}$ & 1.64104947 & 0.61736710 & -2.27617994 \\
$\mathrm{Al}$ & 3.08274101 & 1.15688586 & -1.26385184 \\
$\mathrm{~N}$ & 2.93942439 & 2.50255921 & 0.00000000 \\
$\mathrm{Al}$ & 1.63134949 & -3.39590195 & 0.00000000 \\
$\mathrm{Al}$ & -2.78115933 & 1.50445604 & -1.52959850
\end{tabular}




$\begin{array}{rrrr}\mathrm{N} & -3.25594596 & -0.26977721 & -1.53618944 \\ \mathrm{Al} & -1.78661693 & -0.53305813 & -2.63832761 \\ \mathrm{~N} & -1.38626129 & 1.24335707 & -2.71686566 \\ \mathrm{Al} & -3.40396918 & -1.22409824 & 0.00000000 \\ \mathrm{~N} & -2.54491457 & 2.46448179 & 0.00000000 \\ \mathrm{Al} & 1.51620084 & 3.51947378 & 0.00000000 \\ \mathrm{Al} & 0.26412884 & 1.89570892 & -2.43495026 \\ \mathrm{~N} & 0.27280726 & 3.35499091 & -1.34312883 \\ \mathrm{~N} & -0.04226858 & -3.90763793 & 0.00000000 \\ \mathrm{Al} & -1.12425497 & -3.11677962 & -1.26101522 \\ \mathrm{~N} & -0.52344157 & -1.80686812 & -2.39277466 \\ \mathrm{~N} & -2.41634349 & -2.72793294 & 0.00000000 \\ \mathrm{Al} & -1.00207662 & 3.40677331 & 0.00000000 \\ \mathrm{~N} & 2.35964194 & -2.34144934 & 1.33926034 \\ \mathrm{Al} & 1.26348115 & -1.23713554 & 2.29275237 \\ \mathrm{~N} & 1.64104947 & 0.61736710 & 2.27617994 \\ \mathrm{Al} & 3.08274101 & 1.15688586 & 1.26385184 \\ \mathrm{Al} & -2.78115933 & 1.50445604 & 1.52959850 \\ \mathrm{~N} & -3.25594596 & -0.26977721 & 1.53618944 \\ \mathrm{Al} & -1.78661693 & -0.53305813 & 2.63832761 \\ \mathrm{~N} & -1.38626129 & 1.24335707 & 2.71686566 \\ \mathrm{Al} & 0.26412884 & 1.89570892 & 2.43495026 \\ \mathrm{~N} & 0.27280726 & 3.35499091 & 1.34312883 \\ \mathrm{Al} & -1.12425497 & -3.11677962 & 1.26101522 \\ \mathrm{~N} & -0.52344157 & -1.80686812 & 2.39277466\end{array}$

$\begin{array}{rrrr}(\mathrm{AlN})_{18} / S_{6} & & \\ \mathrm{Al} & 2.46845399 & 2.33319975 & -0.90449571 \\ \mathrm{~N} & 2.54890077 & 2.33554091 & 0.94179018 \\ \mathrm{Al} & -0.45091074 & 2.33319975 & 2.58999172 \\ \mathrm{~N} & -2.09006461 & 2.33554091 & 1.73651773 \\ \mathrm{Al} & -2.01754325 & 2.33319975 & -1.68549601 \\ \mathrm{~N} & -0.45883616 & 2.33554091 & -2.67830791 \\ \mathrm{Al} & 2.55037684 & -0.79101786 & -0.94161328 \\ \mathrm{~N} & 2.54278724 & -0.79061355 & 0.91710710 \\ \mathrm{Al} & -0.45972740 & -0.79101786 & 2.67949778 \\ \mathrm{~N} & -2.06563167 & -0.79061355 & 1.74356480 \\ \mathrm{Al} & -2.09064944 & -0.79101786 & -1.73788449 \\ \mathrm{~N} & -0.47715557 & -0.79061355 & -2.66067190 \\ \mathrm{Al} & 2.09064944 & 0.79101786 & 1.73788449 \\ \mathrm{~N} & 0.47715557 & 0.79061355 & 2.66067190 \\ \mathrm{Al} & -2.55037684 & 0.79101786 & 0.94161328 \\ \mathrm{~N} & -2.54278724 & 0.79061355 & -0.91710710 \\ \mathrm{Al} & 0.45972740 & 0.79101786 & -2.67949778 \\ \mathrm{~N} & 2.06563167 & 0.79061355 & -1.74356480 \\ \mathrm{Al} & 2.01754325 & -2.33319975 & 1.68549601 \\ \mathrm{~N} & 0.45883616 & -2.33554091 & 2.67830791 \\ \mathrm{Al} & -2.46845399 & -2.33319975 & 0.90449571 \\ \mathrm{~N} & -2.54890077 & -2.33554091 & -0.94179018 \\ \mathrm{Al} & 0.45091074 & -2.33319975 & -2.58999172 \\ \mathrm{~N} & 2.09006461 & -2.33554091 & -1.73651773 \\ \mathrm{Al} & 1.56593922 & 3.89253663 & 0.90936294\end{array}$




$\begin{array}{rrrr}\text { Al } & -1.57050102 & 3.89253663 & 0.90146168 \\ \mathrm{~N} & -1.61337275 & 3.95387530 & -0.88513408 \\ \mathrm{Al} & 0.00456180 & 3.89253663 & -1.81082462 \\ \mathrm{~N} & 1.57323497 & 3.95387530 & -0.95465475 \\ \mathrm{~N} & 0.04013778 & 3.95387530 & 1.83978883 \\ \mathrm{Al} & -0.00456180 & -3.89253663 & 1.81082462 \\ \mathrm{Al} & -1.56593922 & -3.89253663 & -0.90936294 \\ \mathrm{~N} & -0.04013778 & -3.95387530 & -1.83978883 \\ \mathrm{Al} & 1.57050102 & -3.89253663 & -0.90146168 \\ \mathrm{~N} & 1.61337275 & -3.95387530 & 0.88513408 \\ \mathrm{~N} & -1.57323497 & -3.95387530 & 0.95465475\end{array}$

\begin{tabular}{rrrr}
$(\mathrm{AlN})$ & \multicolumn{1}{l}{${ }_{19} \mathrm{C}_{3}$} & & \\
$\mathrm{Al}$ & -3.36302272 & 0.69309139 & -1.45479697 \\
$\mathrm{Al}$ & -0.57468326 & 1.78226582 & -2.34875655 \\
$\mathrm{Al}$ & -1.01261139 & 2.93133819 & 1.95986158 \\
$\mathrm{Al}$ & -0.00147805 & 3.94992678 & -0.10623582 \\
$\mathrm{~N}$ & -3.75060498 & 1.52087800 & 0.15729570 \\
$\mathrm{~N}$ & 0.00000000 & 0.00000000 & -2.51949783 \\
$\mathrm{~N}$ & 0.56490332 & 3.04964553 & -1.59041774 \\
$\mathrm{Al}$ & -2.87714040 & 2.98412446 & -0.51009501 \\
$\mathrm{Al}$ & -3.08316020 & 0.63816946 & 1.60307946 \\
$\mathrm{~N}$ & 0.68220804 & 3.43112790 & 1.52982380 \\
$\mathrm{~N}$ & -2.33192670 & 2.07886460 & -2.04764219 \\
$\mathrm{~N}$ & -1.43828917 & 1.19657825 & 2.30216104 \\
$\mathrm{Al}$ & 0.00000000 & 0.00000000 & 2.49539848 \\
$\mathrm{~N}$ & -1.72578137 & 3.85560190 & 0.49938792 \\
$\mathrm{Al}$ & 2.28174611 & 2.56591742 & -1.45479697 \\
$\mathrm{Al}$ & 1.83082911 & -0.39344261 & -2.34875655 \\
$\mathrm{Al}$ & 3.04491904 & -0.58872191 & 1.95986158 \\
$\mathrm{Al}$ & 3.42147595 & -1.97368336 & -0.10623582 \\
$\mathrm{~N}$ & 3.19242148 & 2.48768019 & 0.15729570 \\
$\mathrm{~N}$ & 2.35861884 & -2.01404339 & -1.59041774 \\
$\mathrm{Al}$ & 4.02289779 & 0.99961445 & -0.51009501 \\
$\mathrm{Al}$ & 2.09425107 & 2.35101033 & 1.60307946 \\
$\mathrm{~N}$ & 2.63033990 & -2.30637344 & 1.52982380 \\
$\mathrm{~N}$ & 2.96631290 & 0.98007546 & -2.04764219 \\
$\mathrm{~N}$ & 1.75541175 & 0.64730584 & 2.30216104 \\
$\mathrm{~N}$ & 4.20193988 & -0.43323044 & 0.49938792 \\
$\mathrm{Al}$ & 1.08127661 & -3.25900880 & -1.45479697 \\
$\mathrm{Al}$ & -1.25614584 & -1.38882321 & -2.34875655 \\
$\mathrm{Al}$ & -2.03230765 & -2.34261628 & 1.95986158 \\
$\mathrm{Al}$ & -3.41999791 & -1.97624341 & -0.10623582 \\
$\mathrm{~N}$ & 0.55818350 & -4.00855819 & 0.15729570 \\
$\mathrm{~N}$ & -2.92352217 & -1.03560214 & -1.59041774 \\
$\mathrm{Al}$ & -1.14575739 & -3.98373890 & -0.51009501 \\
$\mathrm{Al}$ & 0.98890914 & -2.98917979 & 1.60307946 \\
$\mathrm{~N}$ & -3.31254794 & -1.12475445 & 1.52982380 \\
$\mathrm{~N}$ & -0.63438620 & -3.05894006 & -2.04764219 \\
$\mathrm{~N}$ & -0.31712258 & -1.84388409 & 2.30216104 \\
$\mathrm{~N}$ & -2.47615851 & -3.42237146 & 0.49938792
\end{tabular}




$\begin{array}{rrrr}(\mathrm{AlN})_{20} / C_{2} & & \\ \mathrm{~N} & 0.31098668 & -0.89231226 & 2.48792672 \\ \mathrm{~N} & 2.64578260 & -1.83248019 & -2.15982789 \\ \mathrm{Al} & 2.57073856 & 2.64407066 & -0.86356699 \\ \mathrm{~N} & -3.28885563 & 0.09125730 & -2.19024737 \\ \mathrm{Al} & 0.91915955 & -2.27814259 & -2.30324944 \\ \mathrm{~N} & -1.24499813 & 3.77192116 & 0.47275445 \\ \mathrm{~N} & -2.53020952 & -2.60607935 & -0.88627846 \\ \mathrm{~N} & -2.64582713 & -1.83242169 & 2.15979657 \\ \mathrm{Al} & -0.91921999 & -2.27813454 & 2.30322594 \\ \mathrm{~N} & -0.43509626 & -3.68292005 & 1.27075508 \\ \mathrm{~N} & 0.87993371 & 2.23533084 & 2.25796417 \\ \mathrm{Al} & -0.35363565 & 0.86595223 & 2.47902077 \\ \mathrm{~N} & 3.28886191 & 0.09114554 & 2.19025918 \\ \mathrm{Al} & 0.41460894 & 3.62050404 & 1.18175235 \\ \mathrm{Al} & 2.10817421 & -1.26500537 & 2.12424034 \\ \mathrm{Al} & -2.10820870 & -1.26492920 & -2.12425384 \\ \mathrm{Al} & 2.61014093 & 1.78180524 & 2.08461382 \\ \mathrm{~N} & -2.13542899 & 1.26779125 & 2.06597079 \\ \mathrm{~N} & 2.13545883 & 1.26774737 & -2.06595131 \\ \mathrm{Al} & -2.57066580 & 2.64413473 & 0.86359483 \\ \mathrm{~N} & 1.24510965 & 3.77190491 & -0.47272585 \\ \mathrm{Al} & -2.61009197 & 1.78189858 & -2.08458768 \\ \mathrm{Al} & -0.41450181 & 3.62052587 & -1.18172369 \\ \mathrm{Al} & 3.28500425 & -0.13092288 & -2.08406365 \\ \mathrm{Al} & -3.28500942 & -0.13085007 & 2.08405838 \\ \mathrm{~N} & -0.87987222 & 2.23537258 & -2.25794076 \\ \mathrm{Al} & -1.17729643 & -3.69278622 & -0.43492073 \\ \mathrm{~N} & 0.43499963 & -3.68292507 & -1.27078959 \\ \mathrm{Al} & 3.52666430 & -2.11453867 & -0.55503705 \\ \mathrm{~N} & 4.44207975 & -0.53156647 & -0.67732168 \\ \mathrm{~N} & -3.59580002 & 2.19303355 & -0.56447072 \\ \mathrm{Al} & -4.35342405 & 0.53645558 & -0.73130749 \\ \mathrm{~N} & 2.53013794 & -2.60615420 & 0.88625091 \\ \mathrm{Al} & 1.17719753 & -3.69282531 & 0.43488629 \\ \mathrm{Al} & -3.52672512 & -2.11444462 & 0.55501111 \\ \mathrm{~N} & -0.31101081 & -0.89228705 & -2.48794114 \\ \mathrm{Al} & 4.35343412 & 0.53632791 & 0.73131548 \\ \mathrm{~N} & 3.59585351 & 2.19292761 & 0.56449859 \\ \mathrm{~N} & 0.35365710 & 0.86596042 & -2.47901110 \\ \mathrm{~N} & -4.44210575 & -0.53145253 & 0.67731931\end{array}$

$\begin{array}{rrrr}(\mathrm{AlN})_{21} / C_{3 \mathrm{~h}} & & \\ \mathrm{~N} & -1.59999447 & -0.92120471 & -2.54034881 \\ \mathrm{Al} & -1.60167563 & 0.92906121 & -2.54353529 \\ \mathrm{~N} & -1.59999447 & 2.66060896 & 0.47238773 \\ \mathrm{Al} & -1.60167563 & 1.73823557 & 2.07635826 \\ \mathrm{~N} & -1.59999447 & -1.73940425 & 2.06796109 \\ \mathrm{Al} & -1.60167563 & -2.66729678 & 0.46717703 \\ \mathrm{~N} & -3.13838640 & 1.74790928 & -2.10489735\end{array}$




$\begin{array}{rrrr}\mathrm{Al} & -3.13185642 & 2.60680126 & -0.46865759 \\ \mathrm{~N} & -3.13838640 & 0.94893994 & 2.56618252 \\ \mathrm{Al} & -3.13185642 & -0.89753125 & 2.49188491 \\ \mathrm{~N} & -3.13838640 & -2.69684922 & -0.46128516 \\ \mathrm{Al} & -3.13185642 & -1.70927000 & -2.02322732 \\ \mathrm{~N} & 1.59999447 & -0.92120471 & -2.54034881 \\ \mathrm{Al} & 1.60167563 & 0.92906121 & -2.54353529 \\ \mathrm{~N} & 1.59999447 & 2.66060896 & 0.47238773 \\ \mathrm{Al} & 1.60167563 & 1.73823557 & 2.07635826 \\ \mathrm{~N} & 1.59999447 & -1.73940425 & 2.06796109 \\ \mathrm{Al} & 1.60167563 & -2.66729678 & 0.46717703 \\ \mathrm{~N} & 0.00000000 & 1.61571804 & -2.00671382 \\ \mathrm{Al} & 0.00000000 & 2.53448407 & -0.40729661 \\ \mathrm{~N} & 0.00000000 & 0.93000612 & 2.40260978 \\ \mathrm{Al} & 0.00000000 & -0.91451282 & 2.39857590 \\ \mathrm{~N} & 0.00000000 & -2.54572416 & -0.39589596 \\ \mathrm{Al} & 0.00000000 & -1.61997125 & -1.99127928 \\ \mathrm{~N} & 3.13838640 & 1.74790928 & -2.10489735 \\ \mathrm{Al} & 3.13185642 & 2.60680126 & -0.46865759 \\ \mathrm{~N} & 3.13838640 & 0.94893994 & 2.56618252 \\ \mathrm{Al} & 3.13185642 & -0.89753125 & 2.49188491 \\ \mathrm{~N} & 3.13838640 & -2.69684922 & -0.46128516 \\ \mathrm{Al} & 3.13185642 & -1.70927000 & -2.02322732 \\ \mathrm{Al} & -4.68442759 & 0.89767052 & -1.57319323 \\ \mathrm{Al} & -4.68442759 & 0.91359004 & 1.56400209 \\ \mathrm{~N} & -4.74306594 & -0.95094692 & 1.57606819 \\ \mathrm{Al} & -4.68442759 & -1.81126056 & 0.00919114 \\ \mathrm{~N} & -4.74306594 & -0.88944163 & -1.61157829 \\ \mathrm{~N} & -4.74306594 & 1.84038855 & 0.03551010 \\ \mathrm{Al} & 4.68442759 & 0.89767052 & -1.57319323 \\ \mathrm{Al} & 4.68442759 & 0.91359004 & 1.56400209 \\ \mathrm{~N} & 4.74306594 & -0.95094692 & 1.57606819 \\ \mathrm{Al} & 4.68442759 & -1.81126056 & 0.00919114 \\ \mathrm{~N} & 4.74306594 & -0.88944163 & -1.61157829 \\ \mathrm{~N} & 4.74306594 & 1.84038855 & 0.03551010\end{array}$

\begin{tabular}{|c|c|c|c|}
\hline \multicolumn{4}{|c|}{$(\mathrm{A} I \mathrm{~N})_{22} / C_{3}$} \\
\hline Al & 0.00133980 & 3.23480517 & -0.35414204 \\
\hline $\mathrm{N}$ & 1.53303200 & 2.66682229 & 0.51072247 \\
\hline Al & 2.63277741 & 1.55263063 & -0.48184831 \\
\hline $\mathrm{N}$ & -0.01522367 & 1.75772415 & -4.27685268 \\
\hline $\mathrm{N}$ & 0.01640197 & 3.34043309 & -2.12734226 \\
\hline Al & 0.00000000 & 0.00000000 & -4.71746627 \\
\hline $\mathrm{N}$ & 2.54269640 & 1.48617990 & -2.33179295 \\
\hline Al & 2.77272595 & -0.14097610 & -3.10912764 \\
\hline Al & 1.25764145 & 2.47314534 & -3.14470082 \\
\hline $\mathrm{N}$ & 3.22498242 & 0.02723636 & 0.36314750 \\
\hline Al & 3.25708012 & 0.04667932 & 2.14388829 \\
\hline $\mathrm{N}$ & 2.52232412 & 1.36360998 & 3.17726180 \\
\hline Al & 1.49241398 & 2.60131783 & 2.36075661 \\
\hline $\mathrm{N}$ & -0.12359230 & 2.85713500 & 3.13526871 \\
\hline Al & 1.75738612 & 0.00318318 & 4.16658399 \\
\hline
\end{tabular}




$\begin{array}{rrrr}\mathrm{N} & 0.00000000 & 0.00000000 & 4.63729194 \\ \mathrm{Al} & 2.80075355 & -1.61856288 & -0.35414204 \\ \mathrm{~N} & 1.54301985 & -2.66105580 & 0.51072247 \\ \mathrm{Al} & 0.02822886 & -3.05636744 & -0.48184831 \\ \mathrm{~N} & 1.52984560 & -0.86567799 & -4.27685268 \\ \mathrm{~N} & 2.88469893 & -1.68442106 & -2.12734226 \\ \mathrm{~N} & 0.01572135 & -2.94512962 & -2.33179295 \\ \mathrm{Al} & -1.50845185 & -2.33076306 & -3.10912764 \\ \mathrm{Al} & 1.51298597 & -2.32572211 & -3.14470082 \\ \mathrm{~N} & -1.58890383 & -2.80653489 & 0.36314750 \\ \mathrm{Al} & -1.58811458 & -2.84405378 & 2.14388829 \\ \mathrm{~N} & -0.08024117 & -2.86620176 & 3.17726180 \\ \mathrm{Al} & 1.50660034 & -2.59312733 & 2.36075661 \\ \mathrm{~N} & 2.53614764 & -1.32153343 & 3.13526871 \\ \mathrm{Al} & -0.87593635 & -1.52353261 & 4.16658399 \\ \mathrm{Al} & -2.80209335 & -1.61624228 & -0.35414204 \\ \mathrm{~N} & -3.07605185 & -0.00576649 & 0.51072247 \\ \mathrm{Al} & -2.66100628 & 1.50373681 & -0.48184831 \\ \mathrm{~N} & -1.51462193 & -0.89204616 & -4.27685268 \\ \mathrm{~N} & -2.90110090 & -1.65601203 & -2.12734226 \\ \mathrm{~N} & -2.55841775 & 1.45894972 & -2.33179295 \\ \mathrm{Al} & -1.26427409 & 2.47173916 & -3.10912764 \\ \mathrm{Al} & -2.77062742 & -0.14742323 & -3.14470082 \\ \mathrm{~N} & -1.63607859 & 2.77929853 & 0.36314750 \\ \mathrm{Al} & -1.66896554 & 2.79737446 & 2.14388829 \\ \mathrm{~N} & -2.44208295 & 1.50259178 & 3.17726180 \\ \mathrm{Al} & -2.99901431 & -0.00819050 & 2.36075661 \\ \mathrm{~N} & -2.41255534 & -1.53560157 & 3.13526871 \\ \mathrm{Al} & -0.88144977 & 1.52034944 & 4.16658399\end{array}$

$\begin{array}{rrrr}(\mathrm{AlN})_{23} / C_{1} & & \\ \mathrm{~N} & 1.95260581 & -1.48064161 & -2.31685681 \\ \mathrm{~N} & 1.61831068 & 3.65615642 & 0.35364508 \\ \mathrm{Al} & 2.10234852 & 0.67431597 & 3.87405949 \\ \mathrm{~N} & -3.84620080 & 1.19583039 & 1.39453915 \\ \mathrm{Al} & -0.09158120 & 3.39313923 & -0.09069292 \\ \mathrm{~N} & -0.13593610 & -2.74237188 & 1.96272798 \\ \mathrm{~N} & -3.48789689 & 2.64735318 & -0.83896554 \\ \mathrm{Al} & -4.63541676 & 1.30027040 & -0.27140273 \\ \mathrm{~N} & -0.76176269 & -1.56815323 & -3.94902623 \\ \mathrm{Al} & 0.94609069 & -0.94015240 & -3.69319810 \\ \mathrm{~N} & 0.46157482 & 0.81163203 & -3.97056530 \\ \mathrm{Al} & -1.25174614 & 0.21111446 & -4.16604335 \\ \mathrm{~N} & 2.16850810 & -3.45931822 & -0.02311145 \\ \mathrm{Al} & 1.18268955 & -2.85364053 & -1.35795927 \\ \mathrm{Al} & 1.47135636 & -3.53579404 & 1.66821861 \\ \mathrm{Al} & 2.96293655 & -0.24981576 & -1.39998719 \\ \mathrm{Al} & 3.54761005 & -2.48447500 & 0.67791411 \\ \mathrm{~N} & -0.64118864 & -2.90208795 & -1.17413296 \\ \mathrm{Al} & -1.29495576 & -2.63256575 & 0.53455203 \\ \mathrm{~N} & 3.06643686 & -2.96771599 & 2.41057872 \\ \mathrm{~N} & 1.34826432 & -0.98342742 & 4.10940653\end{array}$




\begin{tabular}{|c|c|c|c|}
\hline Al & -2.83529395 & -0.23148227 & 1.86445813 \\
\hline Al & -0.15810016 & -1.34275085 & 3.18036553 \\
\hline Al & 2.25656500 & 3.00732305 & 1.94490546 \\
\hline Al & -1.63975475 & -2.47923524 & -2.65475165 \\
\hline $\mathrm{N}$ & -1.23899356 & 0.07261762 & 2.72149128 \\
\hline Al & -2.28542229 & 2.35583859 & -2.16976774 \\
\hline $\mathrm{N}$ & -0.51542470 & 2.74278675 & -1.76441442 \\
\hline Al & 2.94960429 & 2.67124977 & -0.44281058 \\
\hline $\mathrm{N}$ & 3.71407826 & 2.18655342 & 1.13846527 \\
\hline $\mathrm{N}$ & -2.88340810 & -1.71825688 & 0.75579141 \\
\hline Al & -3.98690346 & -1.66946008 & -0.68125612 \\
\hline $\mathrm{N}$ & -4.88728411 & -0.14566715 & -1.24408975 \\
\hline $\mathrm{N}$ & 2.46167851 & 1.50717732 & -1.71386236 \\
\hline Al & 0.90022161 & 1.91334966 & -2.63261095 \\
\hline Al & -3.90804189 & -0.28697793 & -2.79532573 \\
\hline $\mathrm{N}$ & -2.67852623 & 0.94850184 & -3.33770807 \\
\hline $\mathrm{N}$ & 3.69705261 & -0.01945674 & 3.23858479 \\
\hline Al & -0.49378863 & 1.76093294 & 2.60117161 \\
\hline Al & 2.99037000 & -1.67575163 & 3.60463096 \\
\hline $\mathrm{N}$ & -3.33411873 & -1.98745139 & -2.36368947 \\
\hline Al & 4.13146241 & 0.48726875 & 1.54707636 \\
\hline $\mathrm{N}$ & 3.83462211 & -0.75111085 & 0.17116758 \\
\hline Al & -2.86105791 & 2.66096916 & 0.88692316 \\
\hline $\mathrm{N}$ & -1.15931585 & 2.88052058 & 1.31767919 \\
\hline $\mathrm{N}$ & 1.24727054 & 2.05352810 & 3.11014435 \\
\hline \multicolumn{4}{|c|}{$(\mathrm{AlN})_{24} / S_{4}$} \\
\hline $\mathrm{N}$ & 3.54947568 & -1.97739142 & -0.93309025 \\
\hline Al & -1.38586101 & 2.51692889 & 1.93050975 \\
\hline Al & -2.25367873 & 3.27038418 & -0.95608025 \\
\hline $\mathrm{N}$ & 2.64392795 & -1.15130990 & 1.94624975 \\
\hline Al & 3.04795196 & -1.58790028 & -2.64688025 \\
\hline $\mathrm{N}$ & 2.57959614 & 0.06260562 & -3.22530025 \\
\hline Al & 3.44360121 & -0.72232048 & 0.33079975 \\
\hline Al & 2.51687531 & 1.38595497 & -1.93057025 \\
\hline Al & -0.14949712 & 2.62077223 & -3.26583025 \\
\hline $\mathrm{N}$ & -1.82687076 & 2.99332380 & -2.71184025 \\
\hline Al & 1.25473199 & 0.05353081 & -4.44972025 \\
\hline $\mathrm{N}$ & -0.03175345 & 1.33858812 & -4.52062025 \\
\hline $\mathrm{N}$ & -1.00905788 & 3.34620390 & 0.32528975 \\
\hline $\mathrm{N}$ & 1.33858511 & 0.03185390 & 4.52061975 \\
\hline Al & -0.05365122 & 1.25473038 & 4.44972975 \\
\hline Al & 2.62077223 & 0.14949712 & 3.26581975 \\
\hline $\mathrm{N}$ & -0.06253858 & 2.57955136 & 3.22528975 \\
\hline $\mathrm{N}$ & 2.99358386 & 1.82686262 & 2.71186975 \\
\hline Al & 1.58801232 & 3.04783387 & 2.64697975 \\
\hline Al & 3.27037002 & 2.25361957 & 0.95603975 \\
\hline $\mathrm{N}$ & 1.97729768 & 3.54928174 & 0.93314975 \\
\hline $\mathrm{N}$ & 3.34608929 & 1.00899572 & -0.32535025 \\
\hline Al & 0.72229543 & 3.44367313 & -0.33079025 \\
\hline $\mathrm{N}$ & 1.15133173 & 2.64409684 & -1.94628025 \\
\hline $\mathrm{N}$ & -3.54947568 & 1.97739142 & -0.93309025 \\
\hline
\end{tabular}




$\begin{array}{rrrr}\mathrm{Al} & 1.38586101 & -2.51692889 & 1.93050975 \\ \mathrm{Al} & 2.25367873 & -3.27038418 & -0.95608025 \\ \mathrm{~N} & -2.64392795 & 1.15130990 & 1.94624975 \\ \mathrm{Al} & -3.04795196 & 1.58790028 & -2.64688025 \\ \mathrm{~N} & -2.57959614 & -0.06260562 & -3.22530025 \\ \mathrm{Al} & -3.44360121 & 0.72232048 & 0.33079975 \\ \mathrm{Al} & -2.51687531 & -1.38595497 & -1.93057025 \\ \mathrm{Al} & 0.14949712 & -2.62077223 & -3.26583025 \\ \mathrm{~N} & 1.82687076 & -2.99332380 & -2.71184025 \\ \mathrm{Al} & -1.25473199 & -0.05353081 & -4.44972025 \\ \mathrm{~N} & 0.03175345 & -1.33858812 & -4.52062025 \\ \mathrm{~N} & 1.00905788 & -3.34620390 & 0.32528975 \\ \mathrm{~N} & -1.33858511 & -0.03185390 & 4.52061975 \\ \mathrm{Al} & 0.05365122 & -1.25473038 & 4.44972975 \\ \mathrm{Al} & -2.62077223 & -0.14949712 & 3.26581975 \\ \mathrm{~N} & 0.06253858 & -2.57955136 & 3.22528975 \\ \mathrm{~N} & -2.99358386 & -1.82686262 & 2.71186975 \\ \mathrm{Al} & -1.58801232 & -3.04783387 & 2.64697975 \\ \mathrm{Al} & -3.27037002 & -2.25361957 & 0.95603975 \\ \mathrm{~N} & -1.97729768 & -3.54928174 & 0.93314975 \\ \mathrm{~N} & -3.34608929 & -1.00899572 & -0.32535025 \\ \mathrm{Al} & -0.72229543 & -3.44367313 & -0.33079025 \\ \mathrm{~N} & -1.15133173 & -2.64409684 & -1.94628025\end{array}$

\begin{tabular}{rrrr}
\multicolumn{3}{c}{$(\mathrm{AlN})_{25} / C_{3}$} & \\
$\mathrm{~N}$ & 0.00000000 & 0.00000000 & -3.52336029 \\
$\mathrm{Al}$ & 0.00000000 & 0.00000000 & 3.50796418 \\
$\mathrm{~N}$ & -1.55618604 & 0.94668166 & 3.26028687 \\
$\mathrm{Al}$ & 1.64790659 & 2.48542973 & 2.35290244 \\
$\mathrm{Al}$ & -1.37479680 & 2.71377916 & 3.03897105 \\
$\mathrm{~N}$ & 0.21898757 & 3.52120851 & 2.61521149 \\
$\mathrm{~N}$ & -3.51238369 & 0.88883241 & 0.72444152 \\
$\mathrm{Al}$ & -1.33714413 & 3.94719465 & -1.69575361 \\
$\mathrm{~N}$ & -3.00438356 & 3.29008521 & -1.20816737 \\
$\mathrm{Al}$ & 1.55706224 & 3.24938010 & -0.71934821 \\
$\mathrm{Al}$ & -0.66281443 & 4.29321812 & 1.21894189 \\
$\mathrm{~N}$ & -0.02637059 & 4.17913357 & -0.46782327 \\
$\mathrm{~N}$ & -2.30328137 & 3.58513235 & 1.72177835 \\
$\mathrm{Al}$ & -3.27317613 & 2.71118290 & 0.47495047 \\
$\mathrm{~N}$ & -2.95058370 & 0.31047471 & -2.34999280 \\
$\mathrm{Al}$ & -2.78923628 & 2.08052709 & -2.55582124 \\
$\mathrm{~N}$ & -1.20516746 & 2.81568371 & -3.12809576 \\
$\mathrm{Al}$ & 0.26043934 & 1.80529106 & -3.28049490 \\
$\mathrm{~N}$ & 1.59794338 & 0.87435581 & 3.26028687 \\
$\mathrm{Al}$ & 1.32849199 & -2.66984384 & 2.35290244 \\
$\mathrm{Al}$ & 3.03760009 & -0.16628063 & 3.03897105 \\
$\mathrm{~N}$ & 2.93996223 & -1.95025305 & 2.61521149 \\
$\mathrm{~N}$ & 2.52594329 & 2.59739730 & 0.72444152 \\
$\mathrm{Al}$ & 4.08694291 & -0.81559654 & -1.69575361 \\
$\mathrm{~N}$ & 4.35148915 & 0.95682988 & -1.20816737 \\
$\mathrm{Al}$ & 2.03551460 & -2.97314551 & -0.71934821 \\
$\mathrm{Al}$ & 4.04944317 & -1.57259493 & 1.21894189
\end{tabular}




\begin{tabular}{|c|c|c|c|}
\hline $\mathrm{N}$ & 3.63242113 & -2.06672919 & -0.46782327 \\
\hline $\mathrm{N}$ & 4.25645637 & 0.20213400 & 1.72177835 \\
\hline $\mathrm{A} 1$ & 3.98454133 & 1.47906223 & 0.47495047 \\
\hline $\mathrm{N}$ & 1.74417084 & 2.40004308 & -2.34999280 \\
\hline $\mathrm{A} 1$ & 3.19640745 & 1.37528593 & -2.55582124 \\
\hline $\mathrm{N}$ & 3.04103735 & -0.36413622 & -3.12809576 \\
\hline Al & 1.43320825 & -1.12819261 & -3.28049490 \\
\hline $\mathrm{N}$ & -0.04175735 & -1.82103747 & 3.26028687 \\
\hline $\mathrm{Al}$ & -2.97639858 & 0.18441410 & 2.35290244 \\
\hline Al & -1.66280330 & -2.54749853 & 3.03897105 \\
\hline $\mathrm{N}$ & -3.15894980 & -1.57095545 & 2.61521149 \\
\hline $\mathrm{N}$ & 0.98644040 & -3.48622971 & 0.72444152 \\
\hline $\mathrm{A} 1$ & -2.74979878 & -3.13159811 & -1.69575361 \\
\hline $\mathrm{N}$ & -1.34710559 & -4.24691509 & -1.20816737 \\
\hline $\mathrm{A} 1$ & -3.59257684 & -0.27623460 & -0.71934821 \\
\hline $\mathrm{Al}$ & -3.38662874 & -2.72062319 & 1.21894189 \\
\hline $\mathrm{N}$ & -3.60605055 & -2.11240439 & -0.46782327 \\
\hline $\mathrm{N}$ & -1.95317501 & -3.78726635 & 1.72177835 \\
\hline $\mathrm{A} 1$ & -0.71136521 & -4.19024513 & 0.47495047 \\
\hline $\mathrm{N}$ & 1.20641286 & -2.71051779 & -2.34999280 \\
\hline $\mathrm{A} 1$ & -0.40717117 & -3.45581302 & -2.55582124 \\
\hline $\mathrm{N}$ & -1.83586989 & -2.45154749 & -3.12809576 \\
\hline $\mathrm{Al}$ & -1.69364759 & -0.67709845 & -3.28049490 \\
\hline \multicolumn{4}{|c|}{$(\mathrm{ALN})_{26} / C_{2 \mathrm{~h}}$} \\
\hline $\mathrm{A} 1$ & 1.78127776 & 0.00000000 & 2.86946534 \\
\hline $\mathrm{N}$ & 0.91091959 & 1.59537466 & 2.56306638 \\
\hline Al & -0.91698337 & 1.59787805 & 2.55073444 \\
\hline $\mathrm{N}$ & -1.79208815 & 0.00000000 & 2.82236414 \\
\hline $\mathrm{Al}$ & -0.91698337 & -1.59787805 & 2.55073444 \\
\hline $\mathrm{N}$ & 0.91091959 & -1.59537466 & 2.56306638 \\
\hline $\mathrm{Al}$ & -3.49190160 & 2.53034018 & 0.92094823 \\
\hline $\mathrm{N}$ & -4.52465959 & 1.33293646 & 1.76425847 \\
\hline $\mathrm{Al}$ & -3.58337776 & 0.00000000 & 2.62422216 \\
\hline $\mathrm{N}$ & -1.87620706 & 2.92803707 & 1.69770519 \\
\hline Al & -3.49190160 & -2.53034018 & 0.92094823 \\
\hline $\mathrm{N}$ & -1.87620706 & -2.92803707 & 1.69770519 \\
\hline $\mathrm{N}$ & -4.52465959 & -1.33293646 & 1.76425847 \\
\hline Al & -0.88319497 & -4.18246412 & 0.88992899 \\
\hline $\mathrm{N}$ & 0.93193402 & -4.25610319 & 0.96214136 \\
\hline $\mathrm{A} 1$ & 1.87449946 & -2.93477754 & 1.72227638 \\
\hline $\mathrm{A} 1$ & 4.46727023 & -1.26113397 & 1.74125469 \\
\hline $\mathrm{N}$ & 3.57521778 & 0.00000000 & 2.74079275 \\
\hline $\mathrm{N}$ & 3.48400220 & -2.52452754 & 0.93098987 \\
\hline Al & 4.46727023 & 1.26113397 & 1.74125469 \\
\hline $\mathrm{N}$ & 3.48400220 & 2.52452754 & 0.93098987 \\
\hline $\mathrm{A} 1$ & 1.87449946 & 2.93477754 & 1.72227638 \\
\hline Al & -0.88319497 & 4.18246412 & 0.88992899 \\
\hline $\mathrm{N}$ & 0.93193402 & 4.25610319 & 0.96214136 \\
\hline $\mathrm{N}$ & 5.52405408 & 0.00000000 & 0.89392231 \\
\hline $\mathrm{A} I$ & -5.44759283 & 0.00000000 & 0.88490876 \\
\hline $\mathrm{N}$ & 1.79208815 & 0.00000000 & -2.82236414 \\
\hline
\end{tabular}




$\begin{array}{rrrr}\mathrm{Al} & 0.91698337 & 1.59787805 & -2.55073444 \\ \mathrm{~N} & -0.91091959 & 1.59537466 & -2.56306638 \\ \mathrm{~A} I & -1.78127776 & 0.00000000 & -2.86946534 \\ \mathrm{~N} & -0.91091959 & -1.59537466 & -2.56306638 \\ \mathrm{Al} & 0.91698337 & -1.59787805 & -2.55073444 \\ \mathrm{~N} & -3.48400220 & 2.52452754 & -0.93098987 \\ \mathrm{Al} & -4.46727023 & 1.26113397 & -1.74125469 \\ \mathrm{~N} & -3.57521778 & 0.00000000 & -2.74079275 \\ \mathrm{Al} & -1.87449946 & 2.93477754 & -1.72227638 \\ \mathrm{~N} & -3.48400220 & -2.52452754 & -0.93098987 \\ \mathrm{Al} & -1.87449946 & -2.93477754 & -1.72227638 \\ \mathrm{Al} & -4.46727023 & -1.26113397 & -1.74125469 \\ \mathrm{~N} & -0.93193402 & -4.25610319 & -0.96214136 \\ \mathrm{Al} & 0.88319497 & -4.18246412 & -0.88992899 \\ \mathrm{~N} & 1.87620706 & -2.92803707 & -1.69770519 \\ \mathrm{~N} & 4.52465959 & -1.33293646 & -1.76425847 \\ \mathrm{Al} & 3.58337776 & 0.00000000 & -2.62422216 \\ \mathrm{Al} & 3.49190160 & -2.53034018 & -0.92094823 \\ \mathrm{~N} & 4.52465959 & 1.33293646 & -1.76425847 \\ \mathrm{Al} & 3.49190160 & 2.53034018 & -0.92094823 \\ \mathrm{~N} & 1.87620706 & 2.92803707 & -1.69770519 \\ \mathrm{~N} & -0.93193402 & 4.25610319 & -0.96214136 \\ \mathrm{Al} & 0.88319497 & 4.18246412 & -0.88992899 \\ \mathrm{Al} & 5.44759283 & 0.00000000 & -0.88490876 \\ \mathrm{~N} & -5.52405408 & 0.00000000 & -0.89392231\end{array}$

\begin{tabular}{rrrr}
\multicolumn{3}{c}{$(\mathrm{AlN})_{27} / C_{3 \mathrm{v}}$} & \\
$\mathrm{Al}$ & -0.83318561 & 3.18424360 & -1.32445501 \\
$\mathrm{~N}$ & -0.81773430 & 3.19482898 & 0.50128893 \\
$\mathrm{Al}$ & 0.80227122 & 3.18424353 & 1.34340635 \\
$\mathrm{~N}$ & 2.22505652 & 2.43939644 & 0.47474272 \\
$\mathrm{Al}$ & 2.20375609 & 2.43026322 & -1.35095018 \\
$\mathrm{~N}$ & 0.58644981 & 2.43939650 & -2.19825692 \\
$\mathrm{Al}$ & 0.25806365 & 1.16183843 & -3.48286474 \\
$\mathrm{~N}$ & 1.60167002 & -0.03069063 & -3.89388553 \\
$\mathrm{Al}$ & 3.30144762 & 0.46247180 & -3.51251952 \\
$\mathrm{~N}$ & 3.59017563 & 1.70726293 & -2.20085543 \\
$\mathrm{Al}$ & -2.28085793 & 2.52774464 & 1.39821540 \\
$\mathrm{~N}$ & -2.05063718 & 1.93420231 & 3.12767995 \\
$\mathrm{Al}$ & -0.63219319 & 2.57871849 & 4.04991020 \\
$\mathrm{~N}$ & 0.82097706 & 3.19705511 & 3.12293254 \\
$\mathrm{~N}$ & -3.71762204 & 1.93420238 & 0.40838806 \\
$\mathrm{Al}$ & -3.89591540 & 2.57871862 & -1.27408138 \\
$\mathrm{~N}$ & -2.41052749 & 3.19705524 & -2.14850352 \\
$\mathrm{Al}$ & 3.22084744 & 1.16183832 & 1.35021690 \\
$\mathrm{~N}$ & 4.19672957 & -0.03069073 & 0.33934092 \\
$\mathrm{Al}$ & 4.62807088 & 0.46247174 & -1.34844715 \\
$\mathrm{~N}$ & 2.76822714 & 0.73782467 & 3.08563876 \\
$\mathrm{Al}$ & 1.83126377 & 1.96711696 & 4.02841827 \\
$\mathrm{~N}$ & -1.49381728 & 0.73782484 & -3.86687959 \\
$\mathrm{Al}$ & -2.75908171 & 1.96711714 & -3.45964567 \\
$\mathrm{~N}$ & -4.43220978 & 1.58603097 & -2.74926906
\end{tabular}




$\begin{array}{rrrr}\mathrm{N} & 0.43910455 & 1.58603078 & 5.19712918 \\ \mathrm{~N} & 4.61351821 & -0.65974853 & -2.82818666 \\ \mathrm{~N} & -2.26524933 & -2.64981703 & -0.43676580 \\ \mathrm{Al} & -2.14894344 & -2.16223142 & 1.31734885 \\ \mathrm{~N} & -0.63852869 & -2.64981709 & 2.21684453 \\ \mathrm{Al} & 0.90954522 & -2.92156126 & 1.29066569 \\ \mathrm{~N} & 0.75546980 & -3.39976983 & -0.46311944 \\ \mathrm{Al} & -0.73751510 & -2.92156119 & -1.39612402 \\ \mathrm{~N} & -0.54118334 & -2.07370585 & -3.01974751 \\ \mathrm{Al} & 1.16648770 & -1.80963196 & -3.63740827 \\ \mathrm{~N} & 2.47161836 & -2.93281706 & -3.09169970 \\ \mathrm{Al} & 2.26540540 & -3.58810149 & -1.38874269 \\ \mathrm{~N} & -3.10060041 & -0.69677333 & 1.90073523 \\ \mathrm{Al} & -2.49736780 & 0.16147382 & 3.40635885 \\ \mathrm{~N} & -1.53734760 & -0.77604643 & 4.61554271 \\ \mathrm{Al} & -0.48886087 & -2.10634269 & 3.90633789 \\ \mathrm{Al} & -4.16865103 & 0.16147388 & 0.68005517 \\ \mathrm{~N} & -4.81065267 & -0.77604630 & -0.72408102 \\ \mathrm{Al} & -3.70294022 & -2.10634256 & -1.33667308 \\ \mathrm{~N} & 2.44551757 & -2.07370596 & 1.85234926 \\ \mathrm{Al} & 3.77072513 & -1.80963206 & 0.61078971 \\ \mathrm{~N} & 3.87655913 & -2.93281711 & -0.79987082 \\ \mathrm{Al} & 2.33853926 & -1.03913501 & 3.36416897 \\ \mathrm{~N} & 1.07154011 & -1.42375400 & 4.59278201 \\ \mathrm{Al} & -1.93698140 & -1.03913485 & -3.61033269 \\ \mathrm{~N} & -3.60670573 & -1.42375382 & -3.03867061 \\ \mathrm{Al} & -4.78440129 & -0.11973381 & -2.45512759 \\ \mathrm{Al} & 0.01718886 & -0.11973400 & 5.37753212 \\ \mathrm{Al} & 4.13185322 & -2.33336898 & -2.53291563\end{array}$

\begin{tabular}{rrrr}
\multicolumn{3}{c}{$(\mathrm{AlN})_{28} / \mathrm{T}$} & \\
$\mathrm{Al}$ & 2.30196281 & 2.30196281 & 2.30196281 \\
$\mathrm{~N}$ & 2.48680687 & 3.26199032 & 0.74126381 \\
$\mathrm{Al}$ & 1.19477189 & 4.45612222 & 0.39259572 \\
$\mathrm{~N}$ & -2.31196153 & 2.31196153 & 2.31196153 \\
$\mathrm{Al}$ & -3.27365877 & 2.50442324 & 0.75216342 \\
$\mathrm{~N}$ & -4.52498403 & 1.27009309 & 0.41596179 \\
$\mathrm{Al}$ & -2.30196281 & -2.30196281 & 2.30196281 \\
$\mathrm{~N}$ & -2.48680687 & -3.26199032 & 0.74126381 \\
$\mathrm{Al}$ & -1.19477189 & -4.45612222 & 0.39259572 \\
$\mathrm{~N}$ & 2.31196153 & -2.31196153 & 2.31196153 \\
$\mathrm{Al}$ & 3.27365877 & -2.50442324 & 0.75216342 \\
$\mathrm{~N}$ & 4.52498403 & -1.27009309 & 0.41596179 \\
$\mathrm{Al}$ & 2.30196281 & -2.30196281 & -2.30196281 \\
$\mathrm{~N}$ & 2.48680687 & -3.26199032 & -0.74126381 \\
$\mathrm{Al}$ & 1.19477189 & -4.45612222 & -0.39259572 \\
$\mathrm{~N}$ & -2.31196153 & -2.31196153 & -2.31196153 \\
$\mathrm{Al}$ & -3.27365877 & -2.50442324 & -0.75216342 \\
$\mathrm{~N}$ & -4.52498403 & -1.27009309 & -0.41596179 \\
$\mathrm{Al}$ & -2.30196281 & 2.30196281 & -2.30196281 \\
$\mathrm{~N}$ & -2.48680687 & 3.26199032 & -0.74126381 \\
$\mathrm{Al}$ & -1.19477189 & 4.45612222 & -0.39259572
\end{tabular}




$\begin{array}{rrrr}\mathrm{N} & 2.31196153 & 2.31196153 & -2.31196153 \\ \mathrm{Al} & 3.27365877 & 2.50442324 & -0.75216342 \\ \mathrm{~N} & 4.52498403 & 1.27009309 & -0.41596179 \\ \mathrm{~N} & 0.74126381 & 2.48680687 & 3.26199032 \\ \mathrm{~N} & 3.26199032 & 0.74126381 & 2.48680687 \\ \mathrm{~N} & -0.74126381 & -2.48680687 & 3.26199032 \\ \mathrm{~N} & -3.26199032 & -0.74126381 & 2.48680687 \\ \mathrm{~N} & 0.74126381 & -2.48680687 & -3.26199032 \\ \mathrm{~N} & 3.26199032 & -0.74126381 & -2.48680687 \\ \mathrm{~N} & -0.74126381 & 2.48680687 & -3.26199032 \\ \mathrm{~N} & -3.26199032 & 0.74126381 & -2.48680687 \\ \mathrm{Al} & 0.39259572 & 1.19477189 & 4.45612222 \\ \mathrm{Al} & 4.45612222 & 0.39259572 & 1.19477189 \\ \mathrm{Al} & -0.39259572 & -1.19477189 & 4.45612222 \\ \mathrm{Al} & -4.45612222 & -0.39259572 & 1.19477189 \\ \mathrm{Al} & 0.39259572 & -1.19477189 & -4.45612222 \\ \mathrm{Al} & 4.45612222 & -0.39259572 & -1.19477189 \\ \mathrm{Al} & -0.39259572 & 1.19477189 & -4.45612222 \\ \mathrm{Al} & -4.45612222 & 0.39259572 & -1.19477189 \\ \mathrm{Al} & 0.75216342 & -3.27365877 & 2.50442324 \\ \mathrm{Al} & 2.50442324 & 0.75216342 & -3.27365877 \\ \mathrm{Al} & -0.75216342 & 3.27365877 & 2.50442324 \\ \mathrm{Al} & -2.50442324 & -0.75216342 & -3.27365877 \\ \mathrm{Al} & 0.75216342 & 3.27365877 & -2.50442324 \\ \mathrm{Al} & 2.50442324 & -0.75216342 & 3.27365877 \\ \mathrm{Al} & -0.75216342 & -3.27365877 & -2.50442324 \\ \mathrm{Al} & -2.50442324 & 0.75216342 & 3.27365877 \\ \mathrm{~N} & 0.41596179 & -4.52498403 & 1.27009309 \\ \mathrm{~N} & 1.27009309 & 0.41596179 & -4.52498403 \\ \mathrm{~N} & -0.41596179 & 4.52498403 & 1.27009309 \\ \mathrm{~N} & -1.27009309 & -0.41596179 & -4.52498403 \\ \mathrm{~N} & 0.41596179 & 4.52498403 & -1.27009309 \\ \mathrm{~N} & 1.27009309 & -0.41596179 & 4.52498403 \\ \mathrm{~N} & -0.41596179 & -4.52498403 & -1.27009309 \\ \mathrm{~N} & -1.27009309 & 0.41596179 & 4.52498403\end{array}$

\begin{tabular}{rrrr}
\multicolumn{2}{c}{$(\mathrm{AlN})_{29} / \boldsymbol{C}_{\mathbf{s}}$} & & \\
$\mathrm{N}$ & -3.50147209 & -2.93327690 & 0.00000000 \\
$\mathrm{~N}$ & -0.85948379 & -3.26297128 & 1.59471083 \\
$\mathrm{Al}$ & -1.75789708 & -3.42242134 & 0.00000000 \\
$\mathrm{~N}$ & -4.60751896 & -0.47898487 & 0.00000000 \\
$\mathrm{Al}$ & -4.08087547 & -1.70779459 & 1.24984382 \\
$\mathrm{~N}$ & -3.10538516 & -1.30431004 & 2.67461468 \\
$\mathrm{Al}$ & -1.76363015 & -2.51591114 & 3.02271874 \\
$\mathrm{Al}$ & -2.87019538 & 0.48278121 & 3.03121775 \\
$\mathrm{~N}$ & -1.83251365 & 0.87128447 & 4.51477870 \\
$\mathrm{Al}$ & -0.88548490 & -0.50566526 & 5.21898287 \\
$\mathrm{~N}$ & -0.89814302 & -2.21445917 & 4.55229144 \\
$\mathrm{Al}$ & -0.93037656 & 2.43497373 & 4.50877290 \\
$\mathrm{Al}$ & -3.74099582 & 1.10148895 & 0.00000000 \\
$\mathrm{~N}$ & -3.01758774 & 1.65938653 & 1.60628653 \\
$\mathrm{Al}$ & -1.85678537 & 3.08664965 & 1.57177878
\end{tabular}




\begin{tabular}{|c|c|c|c|}
\hline $\mathrm{N}$ & -1.01547893 & 3.55763536 & 3.07549656 \\
\hline $\mathrm{N}$ & -1.00248896 & 3.43333666 & 0.00000000 \\
\hline Al & 3.58567284 & -2.70450629 & 0.00000000 \\
\hline $\mathrm{Al}$ & 0.97603839 & -3.21491291 & 1.59876955 \\
\hline $\mathrm{N}$ & 1.87791278 & -3.31979353 & 0.00000000 \\
\hline Al & 4.51646186 & -0.36804667 & 0.00000000 \\
\hline $\mathrm{N}$ & 4.21511582 & -1.58997977 & 1.32335986 \\
\hline Al & 3.16313447 & -1.19107603 & 2.69130726 \\
\hline $\mathrm{N}$ & 1.84922413 & -2.43959424 & 3.02397857 \\
\hline $\mathrm{N}$ & 2.85187302 & 0.58900530 & 3.06399543 \\
\hline $\mathrm{A} 1$ & 1.83721516 & 0.95541691 & 4.56678245 \\
\hline $\mathrm{N}$ & 0.97464167 & -0.45366632 & 5.30246485 \\
\hline $\mathrm{A} 1$ & 0.92203095 & -2.10803417 & 4.51181323 \\
\hline $\mathrm{N}$ & 0.91910992 & 2.50330333 & 4.59250953 \\
\hline $\mathrm{N}$ & 3.64513442 & 1.21504246 & 0.00000000 \\
\hline Al & 2.92872413 & 1.74742377 & 1.61748880 \\
\hline $\mathrm{N}$ & 1.71890413 & 3.12690939 & 1.56523127 \\
\hline $\mathrm{A} 1$ & 0.82587315 & 3.50653371 & 3.06869154 \\
\hline $\mathrm{Al}$ & 0.83714138 & 3.44942344 & 0.00000000 \\
\hline $\mathrm{N}$ & -0.85948379 & -3.26297128 & -1.59471083 \\
\hline$A l$ & -4.08087547 & -1.70779459 & -1.24984382 \\
\hline $\mathrm{N}$ & -3.10538516 & -1.30431004 & -2.67461468 \\
\hline Al & -1.76363015 & -2.51591114 & -3.02271874 \\
\hline $\mathrm{A} 1$ & -2.87019538 & 0.48278121 & -3.03121775 \\
\hline $\mathrm{N}$ & -1.83251365 & 0.87128447 & -4.51477870 \\
\hline Al & -0.88548490 & -0.50566526 & -5.21898287 \\
\hline $\mathrm{N}$ & -0.89814302 & -2.21445917 & -4.55229144 \\
\hline $\mathrm{Al}$ & -0.93037656 & 2.43497373 & -4.50877290 \\
\hline $\mathrm{N}$ & -3.01758774 & 1.65938653 & -1.60628653 \\
\hline Al & -1.85678537 & 3.08664965 & -1.57177878 \\
\hline $\mathrm{N}$ & -1.01547893 & 3.55763536 & -3.07549656 \\
\hline $\mathrm{A} 1$ & 0.97603839 & -3.21491291 & -1.59876955 \\
\hline $\mathrm{N}$ & 4.21511582 & -1.58997977 & -1.32335986 \\
\hline $\mathrm{A} 1$ & 3.16313447 & -1.19107603 & -2.69130726 \\
\hline $\mathrm{N}$ & 1.84922413 & -2.43959424 & -3.02397857 \\
\hline $\mathrm{N}$ & 2.85187302 & 0.58900530 & -3.06399543 \\
\hline Al & 1.83721516 & 0.95541691 & -4.56678245 \\
\hline $\mathrm{N}$ & 0.97464167 & -0.45366632 & -5.30246485 \\
\hline Al & 0.92203095 & -2.10803417 & -4.51181323 \\
\hline $\mathrm{N}$ & 0.91910992 & 2.50330333 & -4.59250953 \\
\hline $\mathrm{Al}$ & 2.92872413 & 1.74742377 & -1.61748880 \\
\hline $\mathrm{N}$ & 1.71890413 & 3.12690939 & -1.56523127 \\
\hline Al & 0.82587315 & 3.50653371 & -3.06869154 \\
\hline \multicolumn{4}{|c|}{$(\mathrm{AlN})_{30} / C_{2}$} \\
\hline $\mathrm{N}$ & -1.16794100 & 0.66851754 & 4.63381992 \\
\hline $\mathrm{N}$ & 2.05896544 & 5.08450938 & -0.41715148 \\
\hline Al & -2.12942407 & 1.13252221 & 3.17297809 \\
\hline $\mathrm{Al}$ & 2.13131081 & 1.65351824 & -3.64853135 \\
\hline $\mathrm{N}$ & 4.86973963 & 1.00004411 & -0.05840598 \\
\hline $\mathrm{N}$ & 3.57907377 & 3.29163209 & 1.72283070 \\
\hline $\mathrm{N}$ & 3.11378089 & 0.16401327 & 2.33601339 \\
\hline
\end{tabular}




\begin{tabular}{|c|c|c|c|}
\hline Al & 0.62296554 & 1.08618371 & 4.68095640 \\
\hline $\mathrm{N}$ & 1.03755527 & 0.82122804 & -4.76309650 \\
\hline Al & -0.77970836 & 0.98457800 & -4.56691301 \\
\hline Al & 0.30613289 & 4.89422944 & 0.08587620 \\
\hline Al & 4.50290830 & 1.45418780 & -1.79113835 \\
\hline $\mathrm{N}$ & -0.82378835 & 3.73948254 & -0.69749878 \\
\hline $\mathrm{N}$ & 3.33330594 & 0.55285284 & -2.78608703 \\
\hline $\mathrm{N}$ & -1.49865388 & 2.55200940 & 2.19005600 \\
\hline Al & 2.47522904 & 4.68398398 & 1.34417652 \\
\hline $\mathrm{N}$ & -1.42074315 & 1.90490844 & -3.14381791 \\
\hline Al & 0.03435855 & 3.37987991 & 2.79541908 \\
\hline Al & -2.74432554 & 1.07793569 & -2.16998733 \\
\hline Al & -1.88636629 & 2.70511781 & 0.39477615 \\
\hline $\mathrm{N}$ & 1.30427678 & 2.35147352 & 3.64641419 \\
\hline Al & 4.62160219 & 2.76985112 & 0.34872769 \\
\hline $\mathrm{N}$ & 4.29251143 & 3.24220262 & -1.41513364 \\
\hline Al & 2.72691609 & 4.09128494 & -1.75642028 \\
\hline $\mathrm{N}$ & -2.96560019 & 1.45561576 & -0.38170796 \\
\hline Al & -0.28669111 & 3.09203954 & -2.32971057 \\
\hline $\mathrm{N}$ & 1.51127097 & 3.13519996 & -2.75438631 \\
\hline $\mathrm{N}$ & 0.71606781 & 4.72953780 & 1.86027158 \\
\hline Al & 2.84943759 & 1.94367030 & 2.74930179 \\
\hline Al & -3.84391195 & 0.25490359 & 0.70550117 \\
\hline $\mathrm{N}$ & 1.16794100 & -0.66851754 & 4.63381992 \\
\hline $\mathrm{N}$ & -2.05896544 & -5.08450938 & -0.41715148 \\
\hline Al & 2.12942407 & -1.13252221 & 3.17297809 \\
\hline Al & -2.13131081 & -1.65351824 & -3.64853135 \\
\hline $\mathrm{N}$ & -4.86973963 & -1.00004411 & -0.05840598 \\
\hline $\mathrm{N}$ & -3.57907377 & -3.29163209 & 1.72283070 \\
\hline $\mathrm{N}$ & -3.11378089 & -0.16401327 & 2.33601339 \\
\hline Al & -0.62296554 & -1.08618371 & 4.68095640 \\
\hline $\mathrm{N}$ & -1.03755527 & -0.82122804 & -4.76309650 \\
\hline Al & 0.77970836 & -0.98457800 & -4.56691301 \\
\hline Al & -0.30613289 & -4.89422944 & 0.08587620 \\
\hline Al & -4.50290830 & -1.45418780 & -1.79113835 \\
\hline $\mathrm{N}$ & 0.82378835 & -3.73948254 & -0.69749878 \\
\hline $\mathrm{N}$ & -3.33330594 & -0.55285284 & -2.78608703 \\
\hline $\mathrm{N}$ & 1.49865388 & -2.55200940 & 2.19005600 \\
\hline Al & -2.47522904 & -4.68398398 & 1.34417652 \\
\hline $\mathrm{N}$ & 1.42074315 & -1.90490844 & -3.14381791 \\
\hline Al & -0.03435855 & -3.37987991 & 2.79541908 \\
\hline Al & 2.74432554 & -1.07793569 & -2.16998733 \\
\hline Al & 1.88636629 & -2.70511781 & 0.39477615 \\
\hline $\mathrm{N}$ & -1.30427678 & -2.35147352 & 3.64641419 \\
\hline Al & -4.62160219 & -2.76985112 & 0.34872769 \\
\hline $\mathrm{N}$ & -4.29251143 & -3.24220262 & -1.41513364 \\
\hline Al & -2.72691609 & -4.09128494 & -1.75642028 \\
\hline $\mathrm{N}$ & 2.96560019 & -1.45561576 & -0.38170796 \\
\hline Al & 0.28669111 & -3.09203954 & -2.32971057 \\
\hline $\mathrm{N}$ & -1.51127097 & -3.13519996 & -2.75438631 \\
\hline $\mathrm{N}$ & -0.71606781 & -4.72953780 & 1.86027158 \\
\hline Al & -2.84943759 & -1.94367030 & 2.74930179 \\
\hline
\end{tabular}




\begin{tabular}{|c|c|c|c|}
\hline \multicolumn{4}{|c|}{$(\mathrm{A} \perp \mathrm{N})_{31} / C_{3}$} \\
\hline $\mathrm{N}$ & -0.36787050 & 2.95270004 & -3.40712871 \\
\hline $\mathrm{N}$ & 2.48723323 & 3.73824224 & -2.29465412 \\
\hline $\mathrm{N}$ & 3.60640347 & 0.33064616 & 1.77561921 \\
\hline Al & -1.08231939 & 3.44147583 & -1.77390471 \\
\hline $\mathrm{N}$ & 2.50385071 & 2.52335242 & 3.72527570 \\
\hline al & 2.40387322 & 3.65963086 & 2.30601670 \\
\hline $\mathrm{N}$ & 3.80811916 & 0.98004537 & -1.33367138 \\
\hline $\mathrm{N}$ & 0.65248219 & 4.13815881 & 2.63477748 \\
\hline Al & 1.83724294 & 3.93331248 & -0.65285729 \\
\hline $\mathrm{N}$ & 0.00973973 & 3.97213369 & -0.40061357 \\
\hline Al & -0.58711969 & 3.92625798 & 1.33828791 \\
\hline $\mathrm{N}$ & -0.54864680 & 1.73504912 & 4.28261196 \\
\hline $\mathrm{N}$ & 2.89657028 & 3.21315104 & 0.65951228 \\
\hline Al & 3.64092767 & 1.55106662 & 0.40706048 \\
\hline Al & 2.88202651 & 0.80038563 & 3.41246577 \\
\hline Al & 0.74166916 & 2.94440418 & 4.03621045 \\
\hline Al & 3.45835260 & 2.20283988 & -2.62107667 \\
\hline Al & 1.38999123 & 3.21365688 & -3.64698737 \\
\hline Al & 1.81628415 & 0.16239283 & -4.30527414 \\
\hline $\mathrm{N}$ & 2.47300555 & 1.81115535 & -4.12767458 \\
\hline Al & 0.00000000 & 0.00000000 & 4.53961382 \\
\hline $\mathrm{N}$ & 0.00000000 & 0.00000000 & -4.55906452 \\
\hline $\mathrm{N}$ & -2.37317799 & -1.79493522 & -3.40712871 \\
\hline $\mathrm{N}$ & -4.48102936 & 0.28488604 & -2.29465412 \\
\hline $\mathrm{N}$ & -2.08954971 & 2.95791395 & 1.77561921 \\
\hline Al & -2.43924580 & -2.65805400 & -1.77390471 \\
\hline $\mathrm{N}$ & -3.43721265 & 0.90672211 & 3.72527570 \\
\hline Al & -4.37126990 & 0.25199984 & 2.30601670 \\
\hline $\mathrm{N}$ & -2.75280377 & 2.80790525 & -1.33367138 \\
\hline $\mathrm{N}$ & -3.90999175 & -1.50401325 & 2.63477748 \\
\hline Al & -4.32497000 & -0.37555718 & -0.65285729 \\
\hline $\mathrm{N}$ & -3.44483855 & -1.97763199 & -0.40061357 \\
\hline Al & -3.10667931 & -2.47158955 & 1.33828791 \\
\hline $\mathrm{N}$ & -1.22827321 & -1.34266662 & 4.28261196 \\
\hline $\mathrm{N}$ & -4.23095556 & 0.90192793 & 0.65951228 \\
\hline Al & -3.16372693 & 2.37760254 & 0.40706048 \\
\hline Al & -2.13416754 & 2.09571536 & 3.41246577 \\
\hline Al & -2.92076340 & -0.82989776 & 4.03621045 \\
\hline Al & -3.63689160 & 1.89360126 & -2.62107667 \\
\hline Al & -3.47810411 & -0.40306072 & -3.64698737 \\
\hline Al & -1.04877839 & 1.49175180 & -4.30527414 \\
\hline $\mathrm{N}$ & -2.80500932 & 1.23610795 & -4.12767458 \\
\hline $\mathrm{N}$ & 2.74104849 & -1.15776482 & -3.40712871 \\
\hline $\mathrm{N}$ & 1.99379613 & -4.02312828 & -2.29465412 \\
\hline $\mathrm{N}$ & -1.51685377 & -3.28856010 & 1.77561921 \\
\hline Al & 3.52156519 & -0.78342183 & -1.77390471 \\
\hline $\mathrm{N}$ & 0.93336195 & -3.43007453 & 3.72527570 \\
\hline Al & 1.96739668 & -3.91163070 & 2.30601670 \\
\hline $\mathrm{N}$ & -1.05531539 & -3.78795062 & -1.33367138 \\
\hline
\end{tabular}




$\begin{array}{rrrr}\mathrm{N} & 3.25750955 & -2.63414556 & 2.63477748 \\ \mathrm{Al} & 2.48772705 & -3.55775530 & -0.65285729 \\ \mathrm{~N} & 3.43509882 & -1.99450170 & -0.40061357 \\ \mathrm{Al} & 3.69379899 & -1.45466843 & 1.33828791 \\ \mathrm{~N} & 1.77692001 & -0.39238249 & 4.28261196 \\ \mathrm{~N} & 1.33438528 & -4.11507897 & 0.65951228 \\ \mathrm{Al} & -0.47720074 & -3.92866916 & 0.40706048 \\ \mathrm{Al} & -0.74785897 & -2.89610099 & 3.41246577 \\ \mathrm{Al} & 2.17909424 & -2.11450642 & 4.03621045 \\ \mathrm{Al} & 0.17853900 & -4.09644114 & -2.62107667 \\ \mathrm{Al} & 2.08811288 & -2.81059615 & -3.64698737 \\ \mathrm{Al} & -0.76750576 & -1.65414463 & -4.30527414 \\ \mathrm{~N} & 0.33200377 & -3.04726330 & -4.12767458\end{array}$

$\begin{array}{rrrr}(\mathrm{AlN})_{32} / C_{1} & & \\ \mathrm{Al} & 4.63934243 & 1.18668895 & -1.21089195 \\ \mathrm{~N} & 4.70375936 & -0.18498609 & -0.16698711 \\ \mathrm{Al} & 4.21217841 & -1.74651700 & -0.87412396 \\ \mathrm{~N} & 3.13502747 & -1.72869413 & -2.30195711 \\ \mathrm{Al} & 2.75411012 & -0.18590239 & -3.10992158 \\ \mathrm{~N} & 3.81792890 & 1.16654726 & -2.81283590 \\ \mathrm{Al} & 3.02875338 & 2.69154453 & -2.26469297 \\ \mathrm{~N} & 3.93086886 & 2.76406700 & -0.70140951 \\ \mathrm{~N} & 1.10836355 & 0.21184665 & -3.65388277 \\ \mathrm{Al} & 0.38287022 & 1.80818050 & -3.31564813 \\ \mathrm{~N} & -1.38158134 & 2.05891753 & -3.32323068 \\ \mathrm{Al} & -2.30445293 & 0.79924752 & -4.08717643 \\ \mathrm{~N} & -1.60946869 & -0.82942700 & -4.40716045 \\ \mathrm{Al} & 0.01282177 & -1.15623165 & -3.87879677 \\ \mathrm{Al} & 1.93300466 & -3.02388581 & -2.47042770 \\ \mathrm{~N} & 2.13490887 & -4.50199838 & -1.56400665 \\ \mathrm{Al} & 3.18983146 & -4.48091702 & -0.11592250 \\ \mathrm{~N} & 4.26799045 & -3.14957404 & 0.23068547 \\ \mathrm{Al} & 0.80294698 & -4.90116834 & -0.40647979 \\ \mathrm{~N} & 1.87711135 & -5.03100846 & 1.01882671 \\ \mathrm{~N} & 0.27120383 & -2.62433798 & -2.93171086 \\ \mathrm{Al} & -2.97005697 & -1.44565053 & -3.39432787 \\ \mathrm{~N} & -2.65773017 & -2.40430973 & -1.97206471 \\ \mathrm{Al} & -1.06710479 & -3.17433802 & -1.90148817 \\ \mathrm{Al} & 4.32890307 & -2.80786186 & 1.95448871 \\ \mathrm{~N} & 4.45533412 & -1.14814595 & 2.63785250 \\ \mathrm{Al} & 4.30426718 & 0.18597922 & 1.53609994 \\ \mathrm{Al} & 1.53987569 & -3.99448324 & 2.39920841 \\ \mathrm{~N} & 2.99703748 & -3.30339473 & 3.09088592 \\ \mathrm{Al} & 2.92333842 & 2.76750846 & 0.73138876 \\ \mathrm{~N} & 3.12611801 & 1.46853938 & 1.91920005 \\ \mathrm{Al} & 2.99076075 & -1.58443377 & 3.59444011 \\ \mathrm{~N} & 1.56431201 & -0.60829620 & 3.56084900 \\ \mathrm{Al} & 0.03488501 & -1.44325746 & 3.22497938 \\ \mathrm{~N} & -1.40586143 & -0.44456765 & 3.22859135 \\ \mathrm{Al} & -1.27104727 & 1.32237529 & 3.39375503 \\ \mathrm{~N} & 0.30180592 & 2.06027628 & 3.00703803\end{array}$




$\begin{array}{rrrr}\text { Al } & 1.74208762 & 1.05264344 & 2.95735161 \\ \mathrm{~N} & -2.75610005 & 2.31822754 & 3.29947553 \\ \mathrm{Al} & -4.23503846 & 1.50850139 & 2.82074094 \\ \mathrm{~N} & -5.15058670 & 1.92299846 & 1.30432343 \\ \mathrm{Al} & -4.38050036 & 3.04240826 & 0.18460132 \\ \mathrm{~N} & -3.49236792 & 4.31479738 & 0.99390242 \\ \mathrm{Al} & -2.62986097 & 3.89515758 & 2.54015754 \\ \mathrm{~N} & -4.33743654 & -0.26354374 & 2.55116071 \\ \mathrm{~N} & -1.06500822 & 4.56785657 & 1.98949657 \\ \mathrm{Al} & -1.86700134 & 4.74276653 & 0.37552367 \\ \mathrm{~N} & -3.76854568 & 2.41817433 & -1.36593272 \\ \mathrm{Al} & -4.28185308 & 0.76522223 & -1.80056111 \\ \mathrm{~N} & -4.52017696 & -0.47535305 & -0.53540326 \\ \mathrm{Al} & -5.09082157 & 0.15953219 & 0.96791558 \\ \mathrm{~N} & -3.81199958 & 0.14895939 & -3.34819930 \\ \mathrm{~N} & 0.03222053 & -3.04308514 & 2.43489939 \\ \mathrm{Al} & -2.84118326 & -1.14484536 & 2.46003679 \\ \mathrm{Al} & -2.20557535 & 2.97934997 & -2.03718385 \\ \mathrm{Al} & -1.16439874 & -3.24762179 & 1.12833291 \\ \mathrm{~N} & -2.60219639 & -2.23866001 & 1.08322347 \\ \mathrm{~N} & -0.68547482 & -4.02214647 & -0.39435240 \\ \mathrm{Al} & -3.40758132 & -1.86814301 & -0.45645898 \\ \mathrm{Al} & 0.33468045 & 3.53962065 & 2.03178782 \\ \mathrm{~N} & 1.36302824 & 3.57688359 & 0.59479438 \\ \mathrm{~N} & 1.32663104 & 2.98424085 & -2.39055408 \\ \mathrm{Al} & 0.56674196 & 3.74130003 & -0.98330603 \\ \mathrm{~N} & -1.17825852 & 4.05119604 & -1.06326160\end{array}$

\begin{tabular}{rrrr}
\multicolumn{3}{c}{$(\mathrm{AlN}){ }_{33} / \boldsymbol{C}_{\mathbf{s}}$} & \\
$\mathrm{N}$ & 0.99551893 & -3.93452110 & 0.00000000 \\
$\mathrm{Al}$ & -5.56504512 & -1.27588154 & 0.00000000 \\
$\mathrm{Al}$ & 5.59450705 & 2.88890386 & 0.00000000 \\
$\mathrm{~N}$ & 1.85068321 & 2.11393607 & -2.68245843 \\
$\mathrm{~N}$ & -1.75988666 & -3.59323686 & -1.56517765 \\
$\mathrm{Al}$ & -0.85396826 & -3.93650786 & 0.00000000 \\
$\mathrm{Al}$ & -4.46838636 & 3.17029818 & -1.26783936 \\
$\mathrm{Al}$ & -0.82972476 & -3.64452996 & -3.09646809 \\
$\mathrm{~N}$ & 0.99126468 & -3.72229732 & -3.12215100 \\
$\mathrm{Al}$ & 1.88513701 & -3.58508460 & -1.57705856 \\
$\mathrm{~N}$ & -0.90413662 & -2.24188842 & -4.29019617 \\
$\mathrm{Al}$ & 0.84962641 & 3.16078480 & -1.57261504 \\
$\mathrm{~N}$ & -5.75822531 & 2.74892029 & 0.00000000 \\
$\mathrm{~N}$ & 5.67130886 & -1.20477192 & 0.00000000 \\
$\mathrm{Al}$ & 0.92327249 & -2.22255370 & -4.19257349 \\
$\mathrm{Al}$ & -3.21607016 & -2.49058915 & -1.55615982 \\
$\mathrm{~N}$ & -4.14854319 & -2.34547487 & 0.00000000 \\
$\mathrm{~N}$ & 4.40205479 & 3.33839391 & -1.34687028 \\
$\mathrm{~N}$ & 3.30180196 & -2.43566832 & -1.55077013 \\
$\mathrm{Al}$ & 4.23841134 & -2.25243243 & 0.00000000 \\
$\mathrm{~N}$ & -0.94003209 & 0.78151783 & -3.54950216 \\
$\mathrm{~N}$ & 1.85654731 & -0.75629929 & -3.76817382 \\
$\mathrm{Al}$ & 3.33499606 & -1.01004407 & -2.71439284
\end{tabular}




\begin{tabular}{|c|c|c|c|}
\hline $\mathrm{Al}$ & -1.84146497 & -0.80489198 & -3.78907338 \\
\hline $\mathrm{N}$ & -3.31529202 & -1.08473658 & -2.74088609 \\
\hline Al & 5.84501706 & 0.11093936 & -1.26380360 \\
\hline Al & 0.90365189 & 0.80013250 & -3.53699028 \\
\hline $\mathrm{N}$ & -5.91277918 & -0.06858257 & -1.34320639 \\
\hline $\mathrm{Al}$ & -1.90616388 & 2.06756243 & -2.66870360 \\
\hline $\mathrm{N}$ & 4.46807652 & 0.39884698 & -2.40581356 \\
\hline $\mathrm{N}$ & 6.42395680 & 1.33620561 & 0.00000000 \\
\hline $\mathrm{Al}$ & 3.67332616 & 2.07075271 & -2.42611581 \\
\hline Al & -6.39098915 & 1.10719938 & 0.00000000 \\
\hline $\mathrm{A} 1$ & -4.50122948 & 0.26603189 & -2.42286558 \\
\hline $\mathrm{N}$ & -0.94259742 & 3.14734386 & -1.57285051 \\
\hline $\mathrm{N}$ & -3.73668421 & 1.95555876 & -2.41498741 \\
\hline $\mathrm{N}$ & -3.42452919 & 3.97976716 & 0.00000000 \\
\hline Al & 3.32298834 & 3.96604961 & 0.00000000 \\
\hline $\mathrm{A} 1$ & -1.67376384 & 3.72181502 & 0.00000000 \\
\hline $\mathrm{N}$ & 1.56682985 & 3.76011049 & 0.00000000 \\
\hline $\mathrm{N}$ & 1.85068321 & 2.11393607 & 2.68245843 \\
\hline $\mathrm{N}$ & -1.75988666 & -3.59323686 & 1.56517765 \\
\hline $\mathrm{A} 1$ & -4.46838636 & 3.17029818 & 1.26783936 \\
\hline $\mathrm{Al}$ & -0.82972476 & -3.64452996 & 3.09646809 \\
\hline $\mathrm{N}$ & 0.99126468 & -3.72229732 & 3.12215100 \\
\hline $\mathrm{Al}$ & 1.88513701 & -3.58508460 & 1.57705856 \\
\hline $\mathrm{N}$ & -0.90413662 & -2.24188842 & 4.29019617 \\
\hline $\mathrm{A} 1$ & 0.84962641 & 3.16078480 & 1.57261504 \\
\hline Al & 0.92327249 & -2.22255370 & 4.19257349 \\
\hline $\mathrm{A} 1$ & -3.21607016 & -2.49058915 & 1.55615982 \\
\hline $\mathrm{N}$ & 4.40205479 & 3.33839391 & 1.34687028 \\
\hline $\mathrm{N}$ & 3.30180196 & -2.43566832 & 1.55077013 \\
\hline $\mathrm{N}$ & -0.94003209 & 0.78151783 & 3.54950216 \\
\hline $\mathrm{N}$ & 1.85654731 & -0.75629929 & 3.76817382 \\
\hline $\mathrm{Al}$ & 3.33499606 & -1.01004407 & 2.71439284 \\
\hline Al & -1.84146497 & -0.80489198 & 3.78907338 \\
\hline $\mathrm{N}$ & -3.31529202 & -1.08473658 & 2.74088609 \\
\hline Al & 5.84501706 & 0.11093936 & 1.26380360 \\
\hline $\mathrm{Al}$ & 0.90365189 & 0.80013250 & 3.53699028 \\
\hline $\mathrm{N}$ & -5.91277918 & -0.06858257 & 1.34320639 \\
\hline Al & -1.90616388 & 2.06756243 & 2.66870360 \\
\hline $\mathrm{N}$ & 4.46807652 & 0.39884698 & 2.40581356 \\
\hline Al & 3.67332616 & 2.07075271 & 2.42611581 \\
\hline Al & -4.50122948 & 0.26603189 & 2.42286558 \\
\hline $\mathrm{N}$ & -0.94259742 & 3.14734386 & 1.57285051 \\
\hline $\mathrm{N}$ & -3.73668421 & 1.95555876 & 2.41498741 \\
\hline
\end{tabular}

(AlN) ${ }_{34} / C_{3}$

$\begin{array}{rr}\text { Al } & -2.84824000 \\ \mathrm{~N} & -3.97160000 \\ \mathrm{Al} & -4.18664000 \\ \mathrm{~N} & -3.31319000 \\ \mathrm{Al} & -2.94544000 \\ \mathrm{~N} & -1.97859000 \\ \mathrm{Al} & -2.00748000\end{array}$
$-0.73552000$
0.93034000
0.31731000
0.97964000
2. 36048000
2. 95543000
2. 08325000

4.41772000

1.06185000

2.66717000

4.08827000

0.83607000

2. 21239000

3. 77105000 


\begin{tabular}{|c|c|c|c|}
\hline $\mathrm{N}$ & -3.90553000 & 0.26960000 & -1.86638000 \\
\hline Al & -3.04162000 & 1.78066000 & -2.20073000 \\
\hline $\mathrm{N}$ & -0.49622000 & 1.69433000 & 4.64842000 \\
\hline Al & 0.00000000 & 0.00000000 & 4.87632000 \\
\hline $\mathrm{N}$ & -0.62204000 & 4.29436000 & -2.66323000 \\
\hline $\mathrm{Al}$ & -3.86788000 & -0.98462000 & -3.07319000 \\
\hline $\mathrm{Al}$ & -1.17156000 & 3.90555000 & -1.05929000 \\
\hline $\mathrm{N}$ & -2.54865000 & 2.81048000 & -0.83177000 \\
\hline $\mathrm{N}$ & 0.05315000 & 4.02772000 & 0.20492000 \\
\hline Al & -3.99343000 & -0.30870000 & -0.19965000 \\
\hline $\mathrm{N}$ & 0.71512000 & 4.05799000 & 3.10060000 \\
\hline Al & -0.51100000 & 3.88539000 & 1.86699000 \\
\hline $\mathrm{N}$ & 0.61198000 & 2.91985000 & -4.54780000 \\
\hline $\mathrm{N}$ & -2.19596000 & 1.87987000 & -3.77320000 \\
\hline Al & -1.08657000 & 3.18914000 & -3.99559000 \\
\hline Al & -1.71499000 & 0.39684000 & -4.65033000 \\
\hline $\mathrm{N}$ & 0.00000000 & 0.00000000 & -4.91242000 \\
\hline Al & 0.78714000 & 2.83441000 & 4.41772000 \\
\hline Al & 2.06110000 & -2.09889000 & 4.41772000 \\
\hline $\mathrm{N}$ & 2.79150000 & 2.97434000 & 1.06185000 \\
\hline $\mathrm{N}$ & 1.18010000 & -3.90468000 & 1.06185000 \\
\hline Al & 2.36812000 & 3.46709000 & 2.66717000 \\
\hline Al & 1.81853000 & -3.78439000 & 2.66717000 \\
\hline $\mathrm{N}$ & 2.50499000 & 2.37949000 & 4.08827000 \\
\hline $\mathrm{N}$ & 0.80820000 & -3.35913000 & 4.08827000 \\
\hline Al & 3.51695000 & 1.37059000 & 0.83607000 \\
\hline Al & -0.57151000 & -3.73107000 & 0.83607000 \\
\hline $\mathrm{N}$ & 3.54877000 & 0.23579000 & 2.21239000 \\
\hline $\mathrm{N}$ & -1.57018000 & -3.19122000 & 2.21239000 \\
\hline $\mathrm{Al}$ & 2.80789000 & 0.69691000 & 3.77105000 \\
\hline Al & -0.80041000 & -2.78016000 & 3.77105000 \\
\hline $\mathrm{N}$ & 2.18624000 & 3.24749000 & -1.86638000 \\
\hline $\mathrm{N}$ & 1.71928000 & -3.51709000 & -1.86638000 \\
\hline Al & 3.06290000 & 1.74380000 & -2.20073000 \\
\hline Al & -0.02128000 & -3.52445000 & -2.20073000 \\
\hline $\mathrm{N}$ & 1.71544000 & -0.41743000 & 4.64842000 \\
\hline $\mathrm{N}$ & -1.21922000 & -1.27690000 & 4.64842000 \\
\hline $\mathrm{N}$ & 4.03004000 & -1.60848000 & -2.66323000 \\
\hline $\mathrm{N}$ & -3.40800000 & -2.68588000 & -2.66323000 \\
\hline Al & 1.08123000 & 3.84199000 & -3.07319000 \\
\hline Al & 2.78665000 & -2.85737000 & -3.07319000 \\
\hline Al & 3.96809000 & -0.93817000 & -1.05929000 \\
\hline $\mathrm{Al}$ & -2.79652000 & -2.96738000 & -1.05929000 \\
\hline $\mathrm{N}$ & 3.70827000 & 0.80195000 & -0.83177000 \\
\hline $\mathrm{N}$ & -1.15963000 & -3.61243000 & -0.83177000 \\
\hline $\mathrm{N}$ & 3.46154000 & -2.05989000 & 0.20492000 \\
\hline $\mathrm{N}$ & -3.51469000 & -1.96783000 & 0.20492000 \\
\hline Al & 1.72938000 & 3.61276000 & -0.19965000 \\
\hline Al & 2.26406000 & -3.30407000 & -0.19965000 \\
\hline $\mathrm{N}$ & 3.15677000 & -2.64831000 & 3.10060000 \\
\hline $\mathrm{N}$ & -3.87188000 & -1.40969000 & 3.10060000 \\
\hline Al & 3.62035000 & -1.50016000 & 1.8669900 \\
\hline
\end{tabular}




$\begin{array}{rrrr}\text { Al } & -3.10935000 & -2.38524000 & 1.86699000 \\ \mathrm{~N} & 2.22267000 & -1.98991000 & -4.54780000 \\ \mathrm{~N} & -2.83465000 & -0.92993000 & -4.54780000 \\ \mathrm{~N} & 2.72599000 & 0.96182000 & -3.77320000 \\ \mathrm{~N} & -0.53004000 & -2.84169000 & -3.77320000 \\ \mathrm{Al} & 3.30516000 & -0.65357000 & -3.99559000 \\ \mathrm{Al} & -2.21859000 & -2.53557000 & -3.99559000 \\ \mathrm{Al} & 1.20117000 & 1.28680000 & -4.65033000 \\ \mathrm{Al} & 0.51382000 & -1.68365000 & -4.65033000\end{array}$

\section{(AlN) ${ }_{35} / C_{\mathrm{s}}$}

$\begin{array}{rrrr}\text { Al } & 0.91841203 & -4.35436299 & 1.60163617 \\ \mathrm{Al} & 3.26124240 & -2.47675845 & 2.64604438 \\ \mathrm{~N} & -0.91642393 & -4.37355143 & 1.59735485 \\ \mathrm{Al} & -1.82678262 & -3.59050233 & 3.00268714 \\ \mathrm{~N} & 4.31528528 & -3.03493052 & 1.32816045 \\ \mathrm{Al} & -4.24876562 & -3.00751708 & 1.25148786 \\ \mathrm{~N} & -3.25204429 & -2.48969724 & 2.63003369 \\ \mathrm{~N} & -3.63501164 & 0.46581148 & 1.58537263 \\ \mathrm{Al} & 3.62720240 & 0.47261664 & 1.59220425 \\ \mathrm{~N} & 1.82384651 & -3.56951990 & 3.00353665 \\ \mathrm{Al} & 1.81573832 & -0.06934646 & 4.08594659 \\ \mathrm{~N} & 3.09717486 & -0.66731649 & 2.91966317 \\ \mathrm{~N} & -1.80235417 & -0.08910242 & 4.05584251 \\ \mathrm{Al} & -3.08981380 & -0.68120114 & 2.89484633 \\ \mathrm{~N} & 0.94584309 & -1.32564005 & 5.03070541 \\ \mathrm{Al} & -0.89277793 & -1.36098199 & 4.94215070 \\ \mathrm{Al} & 0.88101851 & -3.06580702 & 4.43341836 \\ \mathrm{~N} & -0.93820294 & -3.14457917 & 4.48428163 \\ \mathrm{Al} & -3.03630115 & 2.17454844 & 1.56977163 \\ \mathrm{~N} & 0.92322991 & 1.47943775 & 3.70386743 \\ \mathrm{Al} & -0.90572910 & 1.46436195 & 3.68458992 \\ \mathrm{~N} & 3.04663265 & 2.18655910 & 1.56823683 \\ \mathrm{Al} & 1.72558301 & 2.76132145 & 2.69311512 \\ \mathrm{~N} & -1.72759605 & 2.76047055 & 2.70800677 \\ \mathrm{~N} & -1.80376496 & 5.55880520 & 1.34219620 \\ \mathrm{Al} & -0.94036886 & 4.38947789 & 2.42024246 \\ \mathrm{~N} & 0.90984310 & 4.38674461 & 2.40712381 \\ \mathrm{Al} & 1.74621659 & 5.51722443 & 1.26263868 \\ \mathrm{~N} & 0.85978387 & 6.54558549 & 0.00000000 \\ \mathrm{~N} & 1.80969754 & -4.52499180 & 0.00000000 \\ \mathrm{Al} & -1.80894629 & -4.57011162 & 0.00000000 \\ \mathrm{Al} & 3.56257905 & -4.06648891 & 0.00000000 \\ \mathrm{~N} & -3.57492700 & -4.18123550 & 0.00000000 \\ \mathrm{Al} & 4.82862300 & -1.88723427 & 0.00000000 \\ \mathrm{Al} & -4.32183718 & -0.17013293 & 0.00000000 \\ \mathrm{~N} & 4.30379327 & -0.16836752 & 0.00000000 \\ \mathrm{~N} & -4.93269979 & -1.85901641 & 0.00000000 \\ \mathrm{~A} 1 & -3.28359997 & 3.07947806 & 0.00000000 \\ & 3.28536018 & 3.09416158 & 0.00000000 \\ \mathrm{~A} & -2.79686482 & 4.78815183 & 0.00000000 \\ \mathrm{~N} & 0.91841203 & -4.35436299 & -1.60163617\end{array}$




$\begin{array}{rrrr}\text { Al } & 3.26124240 & -2.47675845 & -2.64604438 \\ \mathrm{~N} & -0.91642393 & -4.37355143 & -1.59735485 \\ \mathrm{Al} & -1.82678262 & -3.59050233 & -3.00268714 \\ \mathrm{~N} & 4.31528528 & -3.03493052 & -1.32816045 \\ \mathrm{Al} & -4.24876562 & -3.00751708 & -1.25148786 \\ \mathrm{~N} & -3.25204429 & -2.48969724 & -2.63003369 \\ \mathrm{~N} & -3.63501164 & 0.46581148 & -1.58537263 \\ \mathrm{Al} & 3.62720240 & 0.47261664 & -1.59220425 \\ \mathrm{~N} & 1.82384651 & -3.56951990 & -3.00353665 \\ \mathrm{Al} & 1.81573832 & -0.06934646 & -4.08594659 \\ \mathrm{~N} & 3.09717486 & -0.66731649 & -2.91966317 \\ \mathrm{~N} & -1.80235417 & -0.08910242 & -4.05584251 \\ \mathrm{Al} & -3.08981380 & -0.68120114 & -2.89484633 \\ \mathrm{~N} & 0.94584309 & -1.32564005 & -5.03070541 \\ \mathrm{Al} & -0.89277793 & -1.36098199 & -4.94215070 \\ \mathrm{Al} & 0.88101851 & -3.06580702 & -4.43341836 \\ \mathrm{~N} & -0.93820294 & -3.14457917 & -4.48428163 \\ \mathrm{Al} & -3.03630115 & 2.17454844 & -1.56977163 \\ \mathrm{~N} & 0.92322991 & 1.47943775 & -3.70386743 \\ \mathrm{Al} & -0.90572910 & 1.46436195 & -3.68458992 \\ \mathrm{~N} & 3.04663265 & 2.18655910 & -1.56823683 \\ \mathrm{Al} & 1.72558301 & 2.76132145 & -2.69311512 \\ \mathrm{~N} & -1.72759605 & 2.76047055 & -2.70800677 \\ \mathrm{~N} & -1.80376496 & 5.55880520 & -1.34219620 \\ \mathrm{Al} & -0.94036886 & 4.38947789 & -2.42024246 \\ \mathrm{~N} & 0.90984310 & 4.38674461 & -2.40712381 \\ \mathrm{Al} & 1.74621659 & 5.51722443 & -1.26263868 \\ \mathrm{Al} & -0.90088426 & 6.45753293 & 0.00000000 \\ \mathrm{~N} & 2.88027522 & 4.82498250 & 0.00000000\end{array}$

\begin{tabular}{rrll}
\multicolumn{3}{c}{$(\mathrm{AlN})_{36} / T_{\mathrm{d}}$} & \\
$\mathrm{Al}$ & -3.14986949 & 0.90864858 & -3.14986949 \\
$\mathrm{~N}$ & -4.01364972 & 1.80208431 & -1.80208431 \\
$\mathrm{Al}$ & -3.14986949 & 3.14986949 & -0.90864858 \\
$\mathrm{~N}$ & -1.80208431 & 4.01364972 & -1.80208431 \\
$\mathrm{Al}$ & -0.90864858 & 3.14986949 & -3.14986949 \\
$\mathrm{~N}$ & -1.80208431 & 1.80208431 & -4.01364972 \\
$\mathrm{Al}$ & -0.88630616 & 5.24993273 & -0.88630616 \\
$\mathrm{Al}$ & -0.88630616 & 0.88630616 & -5.24993273 \\
$\mathrm{Al}$ & -5.24993273 & 0.88630616 & -0.88630616 \\
$\mathrm{Al}$ & 3.14986949 & 0.90864858 & 3.14986949 \\
$\mathrm{~N}$ & 4.01364972 & 1.80208431 & 1.80208431 \\
$\mathrm{Al}$ & 3.14986949 & 3.14986949 & 0.90864858 \\
$\mathrm{~N}$ & 1.80208431 & 4.01364972 & 1.80208431 \\
$\mathrm{Al}$ & 0.90864858 & 3.14986949 & 3.14986949 \\
$\mathrm{~N}$ & 1.80208431 & 1.80208431 & 4.01364972 \\
$\mathrm{Al}$ & 0.88630616 & 5.24993273 & 0.88630616 \\
$\mathrm{Al}$ & 0.88630616 & 0.88630616 & 5.24993273 \\
$\mathrm{Al}$ & 5.24993273 & 0.88630616 & 0.88630616 \\
$\mathrm{~N}$ & -3.16532418 & 0.93822492 & 3.16532418 \\
$\mathrm{Al}$ & -4.04596607 & 1.82322966 & 1.82322966 \\
$\mathrm{~N}$ & -3.16532418 & 3.16532418 & 0.93822492
\end{tabular}




\begin{tabular}{|c|c|c|c|}
\hline Al & -1.82322966 & 4.04596607 & 1.82322966 \\
\hline $\mathrm{N}$ & -0.93822492 & 3.16532418 & 3.16532418 \\
\hline $\mathrm{Al}$ & -1.82322966 & 1.82322966 & 4.04596607 \\
\hline $\mathrm{N}$ & -0.94472691 & 5.33141546 & 0.94472691 \\
\hline $\mathrm{N}$ & -0.94472691 & 0.94472691 & 5.33141546 \\
\hline $\mathrm{N}$ & -5.33141546 & 0.94472691 & 0.94472691 \\
\hline $\mathrm{N}$ & 3.16532418 & 0.93822492 & -3.16532418 \\
\hline Al & 4.04596607 & 1.82322966 & -1.82322966 \\
\hline $\mathrm{N}$ & 3.16532418 & 3.16532418 & -0.93822492 \\
\hline Al & 1.82322966 & 4.04596607 & -1.82322966 \\
\hline $\mathrm{N}$ & 0.93822492 & 3.16532418 & -3.16532418 \\
\hline Al & 1.82322966 & 1.82322966 & -4.04596607 \\
\hline $\mathrm{N}$ & 0.94472691 & 5.33141546 & -0.94472691 \\
\hline $\mathrm{N}$ & 0.94472691 & 0.94472691 & -5.33141546 \\
\hline $\mathrm{N}$ & 5.33141546 & 0.94472691 & -0.94472691 \\
\hline $\mathrm{N}$ & -3.16532418 & -0.93822492 & -3.16532418 \\
\hline Al & -4.04596607 & -1.82322966 & -1.82322966 \\
\hline $\mathrm{N}$ & -3.16532418 & -3.16532418 & -0.93822492 \\
\hline Al & -1.82322966 & -4.04596607 & -1.82322966 \\
\hline $\mathrm{N}$ & -0.93822492 & -3.16532418 & -3.16532418 \\
\hline Al & -1.82322966 & -1.82322966 & -4.04596607 \\
\hline $\mathrm{N}$ & -0.94472691 & -5.33141546 & -0.94472691 \\
\hline $\mathrm{N}$ & -0.94472691 & -0.94472691 & -5.33141546 \\
\hline $\mathrm{N}$ & -5.33141546 & -0.94472691 & -0.94472691 \\
\hline $\mathrm{N}$ & 3.16532418 & -0.93822492 & 3.16532418 \\
\hline Al & 4.04596607 & -1.82322966 & 1.82322966 \\
\hline $\mathrm{N}$ & 3.16532418 & -3.16532418 & 0.93822492 \\
\hline Al & 1.82322966 & -4.04596607 & 1.82322966 \\
\hline $\mathrm{N}$ & 0.93822492 & -3.16532418 & 3.16532418 \\
\hline Al & 1.82322966 & -1.82322966 & 4.04596607 \\
\hline $\mathrm{N}$ & 0.94472691 & -5.33141546 & 0.94472691 \\
\hline $\mathrm{N}$ & 0.94472691 & -0.94472691 & 5.33141546 \\
\hline $\mathrm{N}$ & 5.33141546 & -0.94472691 & 0.94472691 \\
\hline Al & -3.14986949 & -0.90864858 & 3.14986949 \\
\hline $\mathrm{N}$ & -4.01364972 & -1.80208431 & 1.80208431 \\
\hline Al & -3.14986949 & -3.14986949 & 0.90864858 \\
\hline $\mathrm{N}$ & -1.80208431 & -4.01364972 & 1.80208431 \\
\hline Al & -0.90864858 & -3.14986949 & 3.14986949 \\
\hline $\mathrm{N}$ & -1.80208431 & -1.80208431 & 4.01364972 \\
\hline Al & -0.88630616 & -5.24993273 & 0.88630616 \\
\hline Al & -0.88630616 & -0.88630616 & 5.24993273 \\
\hline Al & -5.24993273 & -0.88630616 & 0.88630616 \\
\hline Al & 3.14986949 & -0.90864858 & -3.14986949 \\
\hline $\mathrm{N}$ & 4.01364972 & -1.80208431 & -1.80208431 \\
\hline Al & 3.14986949 & -3.14986949 & -0.90864858 \\
\hline $\mathrm{N}$ & 1.80208431 & -4.01364972 & -1.80208431 \\
\hline Al & 0.90864858 & -3.14986949 & -3.14986949 \\
\hline $\mathrm{N}$ & 1.80208431 & -1.80208431 & -4.01364972 \\
\hline Al & 0.88630616 & -5.24993273 & -0.88630616 \\
\hline Al & 0.88630616 & -0.88630616 & -5.24993273 \\
\hline Al & 5.24993273 & -0.88630616 & -0.88630616 \\
\hline
\end{tabular}




\begin{tabular}{|c|c|c|c|}
\hline \multicolumn{4}{|c|}{$(\mathrm{AlN})_{37} / C_{3}$} \\
\hline Al & -2.30964009 & 3.29314396 & 2.41149058 \\
\hline Al & -1.07588141 & 3.96865846 & -3.66922939 \\
\hline $\mathrm{N}$ & 0.66351307 & 4.39786676 & -3.24743886 \\
\hline Al & -3.14778651 & 1.82908809 & -2.83716085 \\
\hline $\mathrm{N}$ & -2.18993065 & 3.33290890 & -2.41201435 \\
\hline Al & 1.25000447 & 4.04077072 & -1.59736852 \\
\hline $\mathrm{N}$ & -3.85074759 & 0.87353113 & -1.44833424 \\
\hline $\mathrm{N}$ & 2.38540827 & 3.47593346 & 1.60225041 \\
\hline $\mathrm{N}$ & 1.85667284 & 3.03061364 & 4.70083517 \\
\hline $\mathrm{N}$ & 0.00000000 & 0.00000000 & -5.59213120 \\
\hline $\mathrm{N}$ & 0.01013363 & 4.05879222 & -0.24974862 \\
\hline Al & 0.60115320 & 3.89698803 & 1.45461345 \\
\hline Al & -1.71416321 & 3.62188274 & -0.66718908 \\
\hline Al & -3.76206559 & 1.49336038 & 0.25492912 \\
\hline $\mathrm{N}$ & -2.73955255 & 2.95144916 & 0.66848700 \\
\hline Al & -4.22506922 & 1.00077471 & 3.25874812 \\
\hline $\mathrm{N}$ & -0.55624188 & 3.61565723 & 2.84420821 \\
\hline Al & 0.08529857 & 2.99672550 & 4.45622540 \\
\hline $\mathrm{N}$ & -3.34240184 & 2.55859311 & 3.68250650 \\
\hline $\mathrm{N}$ & -2.80615687 & 1.00797455 & -4.45199275 \\
\hline Al & 1.14808275 & 3.30584740 & -4.63238704 \\
\hline Al & -1.22652441 & 1.35408974 & -5.34698957 \\
\hline $\mathrm{N}$ & -0.58708322 & 3.01475684 & -5.16240842 \\
\hline Al & -2.58559556 & 1.61156372 & 5.06466101 \\
\hline $\mathrm{N}$ & -0.81254542 & 1.63274722 & 5.32291326 \\
\hline Al & 0.00000000 & 0.00000000 & 5.57284635 \\
\hline Al & 4.00676637 & 0.35363501 & 2.41149058 \\
\hline Al & -1.69712629 & -3.64677897 & 2.41149058 \\
\hline Al & 3.97489975 & -1.05258860 & -3.66922939 \\
\hline Al & -2.89901834 & -2.91606986 & -3.66922939 \\
\hline $\mathrm{N}$ & 3.47690780 & -2.77355256 & -3.24743886 \\
\hline $\mathrm{N}$ & -4.14042087 & -1.62431420 & -3.24743886 \\
\hline $\mathrm{Al}$ & 3.15793001 & 1.81151904 & -2.83716085 \\
\hline Al & -0.01014350 & -3.64060713 & -2.83716085 \\
\hline $\mathrm{N}$ & 3.98134910 & 0.23008112 & -2.41201435 \\
\hline $\mathrm{N}$ & -1.79141845 & -3.56299003 & -2.41201435 \\
\hline Al & 2.87440786 & -3.10292099 & -1.59736852 \\
\hline $\mathrm{Al}$ & -4.12441233 & -0.93784973 & -1.59736852 \\
\hline $\mathrm{N}$ & 2.68187394 & 2.89807967 & -1.44833424 \\
\hline $\mathrm{N}$ & 1.16887365 & -3.77161080 & -1.44833424 \\
\hline $\mathrm{N}$ & 1.81754254 & -3.80379089 & 1.60225041 \\
\hline $\mathrm{N}$ & -4.20295081 & 0.32785743 & 1.60225041 \\
\hline $\mathrm{N}$ & 1.69625198 & -3.12323267 & 4.70083517 \\
\hline $\mathrm{N}$ & -3.55292482 & 0.09261903 & 4.70083517 \\
\hline $\mathrm{N}$ & 3.50995036 & -2.03817209 & -0.24974862 \\
\hline $\mathrm{N}$ & -3.52008399 & -2.02062013 & -0.24974862 \\
\hline $\mathrm{Al}$ & 3.07431403 & -2.46910796 & 1.45461345 \\
\hline Al & -3.67546723 & -1.42788007 & 1.45461345 \\
\hline Al & 3.99372407 & -0.32643248 & -0.66718908 \\
\hline Al & -2.27956085 & -3.29545026 & -0.66718908 \\
\hline Al & 3.17432082 & 2.51136418 & 0.25492912 \\
\hline
\end{tabular}




$\begin{array}{rrrr}\mathrm{Al} & 0.58774477 & -4.00472456 & 0.25492912 \\ \mathrm{~N} & 3.92580623 & 0.89679753 & 0.66848700 \\ \mathrm{~N} & -1.18625368 & -3.84824669 & 0.66848700 \\ \mathrm{Al} & 2.97923093 & 3.15862992 & 3.25874812 \\ \mathrm{Al} & 1.24583829 & -4.15940464 & 3.25874812 \\ \mathrm{~N} & 3.40937195 & -1.32610902 & 2.84420821 \\ \mathrm{~N} & -2.85313007 & -2.28954821 & 2.84420821 \\ \mathrm{Al} & 2.55259112 & -1.57223347 & 4.45622540 \\ \mathrm{Al} & -2.63788969 & -1.42449202 & 4.45622540 \\ \mathrm{~N} & 3.88700754 & 1.61530835 & 3.68250650 \\ \mathrm{~N} & -0.54460571 & -4.17390145 & 3.68250650 \\ \mathrm{~N} & 2.27601000 & 1.92621586 & -4.45199275 \\ \mathrm{~N} & 0.53014687 & -2.93419041 & -4.45199275 \\ \mathrm{Al} & 2.28890646 & -2.64719253 & -4.63238704 \\ \mathrm{Al} & -3.43698920 & -0.65865488 & -4.63238704 \\ \mathrm{Al} & 1.78593832 & 0.38515643 & -5.34698957 \\ \mathrm{Al} & -0.55941391 & -1.73924617 & -5.34698957 \\ \mathrm{~N} & 2.90439762 & -0.99894944 & -5.16240842 \\ \mathrm{~N} & 2.31731440 & -2.01580740 & -5.16240842 \\ \mathrm{Al} & 2.68845290 & 1.43340958 & 5.06466101 \\ \mathrm{Al} & -0.10285734 & -3.04497330 & 5.06466101 \\ \mathrm{~N} & 1.82027328 & -0.11268863 & 5.32291326 \\ \mathrm{~N} & -1.00772786 & -1.52005859 & 5.32291326\end{array}$

\begin{tabular}{rrrr}
\multicolumn{3}{c}{$(\mathrm{AlN})_{38} / C_{2}$} & \\
$\mathrm{~N}$ & 4.44012130 & 1.62214227 & 0.78848159 \\
$\mathrm{Al}$ & 5.28631022 & 1.22284930 & -0.73082230 \\
$\mathrm{~N}$ & 4.85005349 & 2.00680748 & -2.33915655 \\
$\mathrm{Al}$ & 3.34653681 & 2.99943161 & -2.37358055 \\
$\mathrm{~N}$ & 2.80927682 & 3.77595175 & -0.81593986 \\
$\mathrm{Al}$ & 3.45355450 & 3.15860922 & 0.78716609 \\
$\mathrm{~N}$ & 2.62374742 & 3.59457288 & 2.35389336 \\
$\mathrm{Al}$ & 1.44439324 & 4.95747362 & 2.29264834 \\
$\mathrm{~N}$ & 0.72476695 & 5.48301691 & 0.68284098 \\
$\mathrm{Al}$ & 1.18427405 & 4.60709203 & -0.80111813 \\
$\mathrm{~N}$ & -0.20350958 & 4.89753916 & 3.11013317 \\
$\mathrm{Al}$ & -0.91176721 & 5.17770897 & 1.44748743 \\
$\mathrm{Al}$ & 4.83561725 & 0.29521570 & -3.01747002 \\
$\mathrm{~N}$ & 2.06979602 & 2.57747530 & -3.62244566 \\
$\mathrm{Al}$ & 0.30283688 & 3.03046704 & -3.36776772 \\
$\mathrm{~N}$ & -0.15495985 & 3.91397920 & -1.84098598 \\
$\mathrm{Al}$ & 3.89444584 & 0.21647046 & 1.82716462 \\
$\mathrm{~N}$ & 2.99254333 & 0.58540490 & 3.36942951 \\
$\mathrm{Al}$ & 2.34557109 & 2.29058035 & 3.61357588 \\
$\mathrm{Al}$ & 2.42626306 & 1.11918787 & -4.68891962 \\
$\mathrm{~N}$ & -2.06091204 & 4.00977534 & 0.71154162 \\
$\mathrm{Al}$ & -1.79033556 & 3.61435047 & -1.06616800 \\
$\mathrm{~N}$ & 0.87104304 & 2.51315012 & 4.68606814 \\
$\mathrm{Al}$ & -0.58609265 & 3.41827316 & 4.02014250 \\
$\mathrm{~N}$ & -2.05350072 & 2.43737416 & 3.50599179 \\
$\mathrm{Al}$ & -2.74944789 & 2.72719473 & 1.84162487 \\
$\mathrm{~N}$ & -5.31829307 & 0.47457835 & -1.43009305
\end{tabular}




\begin{tabular}{|c|c|c|c|}
\hline Al & -0.55328755 & 1.12890239 & -5.68076949 \\
\hline Al & -2.32641102 & 0.82857778 & 4.33780957 \\
\hline Al & -4.19896238 & 1.67679388 & -0.70691141 \\
\hline $\mathrm{N}$ & 1.18751121 & 0.59217687 & -5.84452503 \\
\hline Al & 0.44720380 & 1.17018168 & 5.76609926 \\
\hline $\mathrm{N}$ & -1.26014610 & 0.46960714 & 5.74892319 \\
\hline Al & -2.62191845 & 1.79373496 & -3.48550282 \\
\hline $\mathrm{N}$ & -3.45869611 & 0.24342506 & -4.00600995 \\
\hline $\mathrm{N}$ & -3.77025714 & 1.44549659 & 1.06396604 \\
\hline $\mathrm{N}$ & -1.04132924 & 2.20796100 & -4.31707383 \\
\hline $\mathrm{N}$ & -2.98372471 & 2.48015250 & -1.83231813 \\
\hline $\mathrm{N}$ & -4.44012130 & -1.62214227 & 0.78848159 \\
\hline Al & -5.28631022 & -1.22284930 & -0.73082230 \\
\hline $\mathrm{N}$ & -4.85005349 & -2.00680748 & -2.33915655 \\
\hline Al & -3.34653681 & -2.99943161 & -2.37358055 \\
\hline $\mathrm{N}$ & -2.80927682 & -3.77595175 & -0.81593986 \\
\hline Al & -3.45355450 & -3.15860922 & 0.78716609 \\
\hline $\mathrm{N}$ & -2.62374742 & -3.59457288 & 2.35389336 \\
\hline Al & -1.44439324 & -4.95747362 & 2.29264834 \\
\hline $\mathrm{N}$ & -0.72476695 & -5.48301691 & 0.68284098 \\
\hline Al & -1.18427405 & -4.60709203 & -0.80111813 \\
\hline $\mathrm{N}$ & 0.20350958 & -4.89753916 & 3.11013317 \\
\hline Al & 0.91176721 & -5.17770897 & 1.44748743 \\
\hline Al & -4.83561725 & -0.29521570 & -3.01747002 \\
\hline $\mathrm{N}$ & -2.06979602 & -2.57747530 & -3.62244566 \\
\hline Al & -0.30283688 & -3.03046704 & -3.36776772 \\
\hline $\mathrm{N}$ & 0.15495985 & -3.91397920 & -1.84098598 \\
\hline Al & -3.89444584 & -0.21647046 & 1.82716462 \\
\hline $\mathrm{N}$ & -2.99254333 & -0.58540490 & 3.36942951 \\
\hline Al & -2.34557109 & -2.29058035 & 3.61357588 \\
\hline Al & -2.42626306 & -1.11918787 & -4.68891962 \\
\hline $\mathrm{N}$ & 2.06091204 & -4.00977534 & 0.71154162 \\
\hline Al & 1.79033556 & -3.61435047 & -1.06616800 \\
\hline $\mathrm{N}$ & -0.87104304 & -2.51315012 & 4.68606814 \\
\hline Al & 0.58609265 & -3.41827316 & 4.02014250 \\
\hline $\mathrm{N}$ & 2.05350072 & -2.43737416 & 3.50599179 \\
\hline Al & 2.74944789 & -2.72719473 & 1.84162487 \\
\hline $\mathrm{N}$ & 5.31829307 & -0.47457835 & -1.43009305 \\
\hline Al & 0.55328755 & -1.12890239 & -5.68076949 \\
\hline Al & 2.32641102 & -0.82857778 & 4.33780957 \\
\hline Al & 4.19896238 & -1.67679388 & -0.70691141 \\
\hline $\mathrm{N}$ & -1.18751121 & -0.59217687 & -5.84452503 \\
\hline Al & -0.44720380 & -1.17018168 & 5.76609926 \\
\hline $\mathrm{N}$ & 1.26014610 & -0.46960714 & 5.74892319 \\
\hline Al & 2.62191845 & -1.79373496 & -3.48550282 \\
\hline $\mathrm{N}$ & 3.45869611 & -0.24342506 & -4.00600995 \\
\hline $\mathrm{N}$ & 3.77025714 & -1.44549659 & 1.06396604 \\
\hline $\mathrm{N}$ & 1.04132924 & -2.20796100 & -4.31707383 \\
\hline $\mathrm{N}$ & 2.98372471 & -2.48015250 & -1.83231813 \\
\hline
\end{tabular}

\section{(AlN) ${ }_{39} / C_{1}$}

$\begin{array}{llll}\mathrm{N} & -3.14630271 & 2.56775429 & -0.89929655\end{array}$ 


\begin{tabular}{|c|c|c|c|}
\hline Al & 2.19131778 & 3.91110091 & 3.97265334 \\
\hline$A 1$ & 3.73762166 & -1.31772572 & 1.16004300 \\
\hline Al & -0.48451356 & 4.88293189 & 0.13530691 \\
\hline $\mathrm{N}$ & -3.60216158 & -3.42552303 & 3.34560454 \\
\hline $\mathrm{Al}$ & 0.32061487 & -5.06789027 & 1.22758409 \\
\hline $\mathrm{N}$ & 1.40548602 & -5.03080095 & 2.65763781 \\
\hline $\mathrm{N}$ & -1.45731439 & -4.65721630 & 1.44130504 \\
\hline $\mathrm{N}$ & 3.35519555 & -3.10643354 & 1.05812507 \\
\hline$A 1$ & -2.71154984 & 2.72622822 & 0.87078930 \\
\hline $\mathrm{Al}$ & -4.56959451 & -2.91642008 & 1.88339359 \\
\hline$A 1$ & 2.90320011 & -3.96065222 & 2.56497557 \\
\hline $\mathrm{N}$ & 1.00247858 & 5.87507074 & 0.64822655 \\
\hline $\mathrm{N}$ & 3.36604820 & -0.43569769 & 2.71617093 \\
\hline $\mathrm{Al}$ & -4.49091990 & 0.15944182 & 1.45786244 \\
\hline $\mathrm{N}$ & 3.15467138 & 4.93768739 & 2.81573609 \\
\hline$A 1$ & -0.74430017 & -1.52428509 & 4.57441648 \\
\hline Al & 2.38976351 & -1.28658542 & 4.01800561 \\
\hline $\mathrm{N}$ & -3.31262974 & 1.44103036 & 2.03978753 \\
\hline $\mathrm{Al}$ & -2.28563832 & 1.01403714 & 3.50183628 \\
\hline $\mathrm{N}$ & 4.12157009 & 2.34630178 & 1.37579129 \\
\hline $\mathrm{Al}$ & -3.75061507 & -1.67808627 & 3.90519082 \\
\hline$A 1$ & 0.70329828 & 1.25018581 & 4.30864298 \\
\hline$A 1$ & 3.43933060 & 1.37053940 & 2.77268060 \\
\hline $\mathrm{N}$ & 2.27868376 & 2.12685657 & 3.98166682 \\
\hline $\mathrm{N}$ & -0.85165094 & 2.06188237 & 3.81762223 \\
\hline Al & -0.76445794 & 3.72555237 & 3.08287265 \\
\hline $\mathrm{N}$ & -0.68223407 & -3.33137505 & 4.23481850 \\
\hline $\mathrm{Al}$ & 0.95158958 & -4.04590062 & 4.14101683 \\
\hline $\mathrm{N}$ & -4.87314511 & -1.21904406 & 2.53341567 \\
\hline $\mathrm{N}$ & 2.50207088 & -3.06993115 & 4.11666018 \\
\hline $\mathrm{N}$ & 0.78665359 & -0.54394018 & 4.51317226 \\
\hline $\mathrm{N}$ & -1.43979418 & 3.93690551 & 1.38930685 \\
\hline $\mathrm{N}$ & 0.59893322 & 4.76369315 & 3.60249371 \\
\hline $\mathrm{N}$ & -2.32595305 & -0.65206546 & 4.26979208 \\
\hline Al & 4.10455010 & 4.20179863 & 1.46984105 \\
\hline Al & -1.95525609 & -4.07514496 & 3.12485791 \\
\hline $\mathrm{Al}$ & 1.52904895 & 5.72425775 & 2.36622379 \\
\hline $\mathrm{N}$ & 2.75245352 & -0.07942969 & -3.32241765 \\
\hline $\mathrm{N}$ & 3.07113809 & -2.90590630 & -2.02482862 \\
\hline Al & 3.82488199 & 4.21642294 & -1.66731645 \\
\hline Al & 2.71373251 & 1.70205222 & -3.02584592 \\
\hline $\mathrm{Al}$ & 0.89548321 & 4.21326958 & -2.66036706 \\
\hline Al & -2.45927302 & -3.18637767 & -3.07191619 \\
\hline $\mathrm{N}$ & -1.43665963 & -2.71516600 & -4.53612278 \\
\hline Al & -3.22314588 & 1.50968979 & -4.36373208 \\
\hline$A 1$ & 0.01221845 & -4.77766522 & -1.87321461 \\
\hline $\mathrm{N}$ & -3.88419855 & -2.08987665 & -2.71176967 \\
\hline$A 1$ & 1.62857912 & -0.76970365 & -4.59111364 \\
\hline $\mathrm{N}$ & -4.53023082 & 0.97274513 & -3.20010728 \\
\hline $\mathrm{N}$ & 0.02803174 & 0.06800565 & -4.89893453 \\
\hline $\mathrm{N}$ & 1.22592731 & 2.63194785 & -3.51484806 \\
\hline $\mathrm{N}$ & 0.83970379 & -4.63306847 & -3.45245846 \\
\hline
\end{tabular}




$\begin{array}{rrrr}\mathrm{Al} & 2.34663968 & -3.57175997 & -3.52130130 \\ \mathrm{~N} & 2.32506359 & 5.16497695 & -2.16792910 \\ \mathrm{Al} & 0.14206052 & -3.52858193 & -4.72393187 \\ \mathrm{~N} & -3.04917809 & -0.15198347 & -5.11641421 \\ \mathrm{Al} & -4.23328589 & -0.73168964 & -3.83465996 \\ \mathrm{~N} & 1.68653458 & -2.53787384 & -4.88421547 \\ \mathrm{Al} & -1.46625048 & -0.97446136 & -5.14860863 \\ \mathrm{Al} & -0.17116704 & 1.76365072 & -4.25701525 \\ \mathrm{~N} & -1.81148344 & 2.43918241 & -3.77505285 \\ \mathrm{Al} & -4.36605675 & -1.83532057 & -0.97119600 \\ \mathrm{~N} & -1.72890157 & -4.19660424 & -1.71332612 \\ \mathrm{~N} & 3.83879048 & 2.43046712 & -1.78748267 \\ \mathrm{Al} & 3.44165433 & -1.10972604 & -1.97290734 \\ \mathrm{Al} & 2.38862292 & 5.92204234 & -0.51339214 \\ \mathrm{~N} & 4.00132298 & 5.09942121 & -0.08435940 \\ \mathrm{Al} & -1.96467002 & 3.24181226 & -2.12513838 \\ \mathrm{~N} & -0.59862973 & 4.30788363 & -1.59509220 \\ \mathrm{Al} & -2.46139370 & -4.17278079 & -0.02983888 \\ \mathrm{~N} & -3.98522158 & -3.17244749 & 0.21082464 \\ \mathrm{~N} & 1.07246542 & -4.73175476 & -0.39361494 \\ \mathrm{Al} & 2.62676675 & -3.76206080 & -0.47817251 \\ \mathrm{~N} & 3.89531608 & -0.33157424 & -0.37243660 \\ \mathrm{Al} & 4.14457279 & 1.47671888 & -0.25147186 \\ \mathrm{~N} & -4.62084528 & -0.13455663 & -0.33323000 \\ \mathrm{Al} & -4.31838495 & 1.27056253 & -1.44855660\end{array}$

$\begin{array}{rrrr}(\mathrm{AlN})_{40} / C_{3} & & \\ \mathrm{~N} & 0.00000000 & 0.00000000 & -6.09961630 \\ \mathrm{Al} & -2.97362945 & 1.84500417 & -5.17854059 \\ \mathrm{~N} & 3.49233602 & 2.78606775 & -3.81066865 \\ \mathrm{Al} & 1.90212472 & 3.64333544 & -4.18469965 \\ \mathrm{~N} & 1.60331789 & 2.61463199 & -5.68219268 \\ \mathrm{Al} & -1.07296262 & 3.47088655 & -3.33433637 \\ \mathrm{Al} & 0.01032483 & 1.82557875 & -5.85371974 \\ \mathrm{~N} & 0.65288392 & 3.93011921 & -2.91767792 \\ \mathrm{~N} & -1.38361533 & 2.64332135 & -4.95769351 \\ \mathrm{~N} & -2.22963084 & 3.22906757 & -1.93595241 \\ \mathrm{al} & -3.67442089 & 2.11930348 & -2.16765892 \\ \mathrm{~N} & 0.00412664 & 3.91992286 & 0.20086037 \\ \mathrm{~N} & 2.80094571 & 3.00292610 & -0.84542901 \\ \mathrm{Al} & 1.20462780 & 3.82142024 & -1.17080972 \\ \mathrm{Al} & -1.72949950 & 3.51401570 & -0.20226819 \\ \mathrm{Al} & 0.57065564 & 3.86985831 & 1.93382278 \\ \mathrm{~N} & -2.77654362 & 2.93351181 & 1.17671341 \\ \mathrm{Al} & 0.06294445 & 2.99891887 & 4.95888301 \\ \mathrm{Al} & -2.59066191 & 1.58998571 & 5.58847209 \\ \mathrm{~N} & -3.36136062 & 2.52383746 & 4.20130567 \\ \mathrm{~N} & 1.82899015 & 3.04659475 & 5.25118881 \\ \mathrm{Al} & 3.18461439 & 2.57916412 & 0.85174979 \\ \mathrm{~N} & 2.35163795 & 3.50907679 & 2.17278346 \\ \mathrm{~N} & -0.58318210 & 3.60488846 & 3.33644192 \\ \mathrm{Al} & 2.94922633 & 3.20151997 & 3.81951801\end{array}$




\begin{tabular}{|c|c|c|c|}
\hline Al & -2.33994284 & 3.27266194 & 2.92128339 \\
\hline $\mathrm{N}$ & -0.81906123 & 1.62799852 & 5.82870095 \\
\hline Al & 0.00000000 & 0.00000000 & 6.07886046 \\
\hline Al & -0.11100575 & -3.49774073 & -5.17854059 \\
\hline Al & 3.08463521 & 1.65273657 & -5.17854059 \\
\hline $\mathrm{N}$ & -4.15897346 & 1.63141784 & -3.81066865 \\
\hline $\mathrm{N}$ & 0.66663744 & -4.41748559 & -3.81066865 \\
\hline Al & -4.10628341 & -0.17437939 & -4.18469965 \\
\hline Al & 2.20415869 & -3.46895605 & -4.18469965 \\
\hline $\mathrm{N}$ & -3.06599667 & 0.08119803 & -5.68219268 \\
\hline $\mathrm{N}$ & 1.46267878 & -2.69583002 & -5.68219268 \\
\hline Al & -2.46939462 & -2.66465617 & -3.33433637 \\
\hline Al & 3.54235724 & -0.80623039 & -3.33433637 \\
\hline Al & -1.58615998 & -0.90384781 & -5.85371974 \\
\hline Al & 1.57583516 & -0.92173094 & -5.85371974 \\
\hline $\mathrm{N}$ & -3.73002503 & -1.39964554 & -2.91767792 \\
\hline $\mathrm{N}$ & 3.07714111 & -2.53047366 & -2.91767792 \\
\hline $\mathrm{N}$ & -1.59737578 & -2.51990670 & -4.95769351 \\
\hline $\mathrm{N}$ & 2.98099110 & -0.12341465 & -4.95769351 \\
\hline $\mathrm{N}$ & -1.68163913 & -3.54545074 & -1.93595241 \\
\hline $\mathrm{N}$ & 3.91126997 & 0.31638316 & -1.93595241 \\
\hline Al & 0.00183979 & -4.24179357 & -2.16765892 \\
\hline Al & 3.67258110 & 2.12249009 & -2.16765892 \\
\hline $\mathrm{N}$ & -3.39681610 & -1.95638765 & 0.20086037 \\
\hline $\mathrm{N}$ & 3.39268946 & -1.96353521 & 0.20086037 \\
\hline $\mathrm{N}$ & -4.00108314 & 0.92422708 & -0.84542901 \\
\hline $\mathrm{N}$ & 1.20013744 & -3.92715319 & -0.84542901 \\
\hline Al & -3.91176091 & -0.86747185 & -1.17080972 \\
\hline Al & 2.70713311 & -2.95394840 & -1.17080972 \\
\hline Al & -2.17847712 & -3.25479836 & -0.20226819 \\
\hline Al & 3.90797662 & -0.25921735 & -0.20226819 \\
\hline Al & -3.63672342 & -1.44072687 & 1.93382278 \\
\hline Al & 3.06606779 & -2.42913144 & 1.93382278 \\
\hline $\mathrm{N}$ & -1.15222394 & -3.87131322 & 1.17671341 \\
\hline $\mathrm{N}$ & 3.92876756 & 0.93780141 & 1.17671341 \\
\hline Al & -2.62861215 & -1.44494795 & 4.95888301 \\
\hline Al & 2.56566770 & -1.55397093 & 4.95888301 \\
\hline Al & -0.08163706 & -3.03857189 & 5.58847209 \\
\hline Al & 2.67229897 & 1.44858617 & 5.58847209 \\
\hline $\mathrm{N}$ & -0.50502705 & -4.17294242 & 4.20130567 \\
\hline $\mathrm{N}$ & 3.86638767 & 1.64910496 & 4.20130567 \\
\hline $\mathrm{N}$ & -3.55292352 & 0.06065456 & 5.25118881 \\
\hline $\mathrm{N}$ & 1.72393337 & -3.10724931 & 5.25118881 \\
\hline Al & -3.82592884 & 1.46837490 & 0.85174979 \\
\hline Al & 0.64131445 & -4.04753902 & 0.85174979 \\
\hline $\mathrm{N}$ & -4.21476861 & 0.28203981 & 2.17278346 \\
\hline $\mathrm{N}$ & 1.86313067 & -3.79111660 & 2.17278346 \\
\hline $\mathrm{N}$ & -2.83033393 & -2.30749474 & 3.33644192 \\
\hline $\mathrm{N}$ & 3.41351603 & -1.29739372 & 3.33644192 \\
\hline Al & -4.24721079 & 0.95334494 & 3.81951801 \\
\hline Al & 1.29798445 & -4.15486491 & 3.81951801 \\
\hline Al & -1.66423696 & -3.66278091 & 2.9212833 \\
\hline
\end{tabular}




$\begin{array}{rrrr}\text { Al } & 4.00417980 & 0.39011898 & 2.92128339 \\ \mathrm{~N} & -1.00035746 & -1.52332710 & 5.82870095 \\ \mathrm{~N} & 1.81941870 & -0.10467143 & 5.82870095\end{array}$

\begin{tabular}{|c|c|c|c|}
\hline \multicolumn{4}{|c|}{$(\mathrm{A} I N)_{41} / C_{1}$} \\
\hline Al & 3.20606976 & 3.26379460 & 3.58728260 \\
\hline $\mathrm{N}$ & 3.00983665 & 1.73644861 & 4.58903929 \\
\hline $\mathrm{Al}$ & 4.49669375 & 1.10438273 & 3.72031485 \\
\hline $\mathrm{N}$ & 4.80632053 & 2.72022327 & 2.88700458 \\
\hline $\mathrm{N}$ & 4.50562920 & -0.45503541 & 2.81366834 \\
\hline Al & 3.05211104 & -1.55395339 & 3.08575776 \\
\hline $\mathrm{N}$ & 2.87415839 & -3.04006850 & 2.03983333 \\
\hline Al & 5.38940757 & -0.47808320 & 1.20301299 \\
\hline Al & 5.07524425 & 2.67166741 & 1.12674049 \\
\hline $\mathrm{N}$ & 5.87854881 & 1.14718838 & 0.47921678 \\
\hline Al & 1.47207777 & 0.84175632 & 4.46076346 \\
\hline $\mathrm{N}$ & 1.59323767 & -0.92384413 & 3.98440769 \\
\hline Al & 4.13275720 & -3.33952823 & 0.73060966 \\
\hline $\mathrm{N}$ & 5.17355653 & -1.94652181 & 0.13851085 \\
\hline $\mathrm{Al}$ & 6.26009082 & 1.17339864 & -1.26946564 \\
\hline $\mathrm{N}$ & 6.08617044 & -0.28615498 & -2.34291012 \\
\hline $\mathrm{Al}$ & 5.39405647 & -1.78884685 & -1.68562435 \\
\hline $\mathrm{N}$ & 3.79478169 & -4.66255343 & -0.44673052 \\
\hline $\mathrm{Al}$ & 2.07851839 & -5.28473093 & -0.62899990 \\
\hline $\mathrm{N}$ & 0.75260602 & -4.82739695 & 0.47173374 \\
\hline Al & 1.23308940 & -3.85314993 & 1.94984393 \\
\hline $\mathrm{N}$ & 4.17274233 & -2.61414933 & -2.77927785 \\
\hline Al & 3.68562910 & -4.23611422 & -2.24044731 \\
\hline $\mathrm{N}$ & 1.99949479 & -4.89639439 & -2.43123728 \\
\hline Al & 0.01293042 & -1.79746848 & 3.94946780 \\
\hline $\mathrm{N}$ & -0.17220093 & -3.31229888 & 2.95787854 \\
\hline $\mathrm{N}$ & 1.87002437 & 3.87209932 & 2.55123607 \\
\hline Al & 0.17583868 & 3.29008791 & 2.96330855 \\
\hline $\mathrm{N}$ & -0.01625304 & 1.78725426 & 3.96878785 \\
\hline Al & 2.29327791 & 4.14365299 & 0.78027728 \\
\hline $\mathrm{N}$ & 3.86096066 & 3.54645995 & 0.05775994 \\
\hline Al & 2.90301755 & -1.58443707 & -3.62226372 \\
\hline $\mathrm{N}$ & 1.15701154 & -2.12484672 & -3.63961992 \\
\hline Al & 0.70216241 & -3.76179893 & -2.93375899 \\
\hline Al & -0.86803511 & -4.47086255 & -0.30555789 \\
\hline $\mathrm{N}$ & -0.86861161 & -3.86959649 & -2.02701292 \\
\hline $\mathrm{N}$ & 0.87269120 & 4.49254008 & -0.28690640 \\
\hline Al & -0.18906026 & -0.89446812 & -3.81135989 \\
\hline $\mathrm{N}$ & -1.93041000 & -1.40944328 & -3.59801306 \\
\hline Al & -2.27834123 & -2.89957437 & -2.58800652 \\
\hline $\mathrm{N}$ & 3.27771789 & 0.19887667 & -3.79877163 \\
\hline $\mathrm{N}$ & 0.19162067 & 0.90091774 & -3.80975229 \\
\hline $\mathrm{N}$ & -2.91057912 & 1.58141875 & -3.64328211 \\
\hline Al & -1.86273520 & -3.89018154 & 2.55064025 \\
\hline Al & -3.84449592 & -3.52993322 & 0.06848075 \\
\hline $\mathrm{N}$ & -2.28522349 & -4.14989598 & 0.78074100 \\
\hline Al & 0.86183926 & 3.87027556 & -2.00344958 \\
\hline
\end{tabular}




$\begin{array}{rrrr}\mathrm{N} & 2.28067809 & 2.92202174 & -2.58576739 \\ \mathrm{Al} & -1.16167798 & 2.11927712 & -3.62925770 \\ \mathrm{~N} & -0.71348672 & 3.75055764 & -2.91169589 \\ \mathrm{~N} & -3.19856001 & -3.36746610 & 3.63136865 \\ \mathrm{Al} & 1.92811127 & 1.42009589 & -3.56866609 \\ \mathrm{~N} & 5.27819641 & 2.25427771 & -2.38744480 \\ \mathrm{Al} & 3.87599963 & 3.15159276 & -1.73291034 \\ \mathrm{Al} & 4.94436826 & 0.73674784 & -3.37294095 \\ \mathrm{~N} & -5.06491552 & -2.66113487 & 1.13391620 \\ \mathrm{~N} & -3.86974608 & -3.12400123 & -1.71684129 \\ \mathrm{~N} & -5.39673793 & 0.48184233 & 1.21612330 \\ \mathrm{Al} & -4.17980092 & 2.61225880 & -2.79234042 \\ \mathrm{Al} & -3.28503172 & -0.20117708 & -3.83592126 \\ \mathrm{Al} & -2.03293117 & 4.82286763 & -2.34136941 \\ \mathrm{Al} & -5.24286796 & -2.16076388 & -2.33644235 \\ \mathrm{Al} & -3.72295160 & 4.60517049 & -0.49291156 \\ \mathrm{~N} & -4.96399461 & -0.74435741 & -3.47645437 \\ \mathrm{~N} & -6.36299969 & -1.20476153 & -1.23120276 \\ \mathrm{~N} & -4.11150509 & 3.32309385 & 0.71717631 \\ \mathrm{Al} & -6.00243122 & 0.22576547 & -2.29970393 \\ \mathrm{~N} & -2.06685320 & 5.38567794 & -0.58774448 \\ \mathrm{Al} & -0.74955994 & 4.84019555 & 0.48359038 \\ \mathrm{Al} & -5.88698448 & -1.14542602 & 0.49326529 \\ \mathrm{Al} & -5.16353678 & 1.93487798 & 0.13324027 \\ \mathrm{~N} & -5.38528263 & 1.77794001 & -1.68675763 \\ \mathrm{~N} & -3.76802530 & 4.27370841 & -2.30954379 \\ \mathrm{Al} & -3.01742440 & -1.74562372 & 4.47946117 \\ \mathrm{Al} & -4.71315962 & -2.66616304 & 2.88104674 \\ \mathrm{Al} & -2.85514669 & 3.03958968 & 2.03699376 \\ \mathrm{~N} & -1.47633884 & -0.84877446 & 4.44514902 \\ \mathrm{~N} & -3.06803922 & 1.56228786 & 3.08790225 \\ \mathrm{~N} & -4.57033004 & -1.06248136 & 3.78141858 \\ \mathrm{Al} & -4.52392506 & 0.46846117 & 2.83359866 \\ \mathrm{Al} & -1.60012641 & 0.92121866 & 3.97546324 \\ \mathrm{~N} & -1.22301564 & 3.86304905 & 1.96082178\end{array}$

